\title{
The Role of $\boldsymbol{\beta}$-catenin in Embryonic Stem Cell Maintenance Circuits and iPSCs - An International Systems Biology Approach of Open Science and Innovation
}

\author{
Roman Anton* \\ Department of Molecular Biology and Biomedicine, Institute of Regenerative \\ Medicine and Stem Cell Medicine The University of Truth and Common Sense, \\ Germany
}

*Corresponding author: Roman Anton, Department of Molecular Biology and Biomedicine, Institute of Regenerative Medicine and Stem Cell Medicine, The University of Truth and Common Sense, Germany, Tel: + 491729143291; Email: mail.roman.anton@gmail.com

\section{Abstract}

Embryonic stem cells (ESCs) and induced pluripotent stem cell (iPSCs), i.e. somatic body cells reprogrammed into ESCs, are the most powerful sources of regenerative medicine. Pluripotency of iPSCs and ESCs bears the capacity to enable all possible cell and organ replacement strategies for regenerative and rejuvenating medicine. This cellular ability is unique and maintained under self-renewal culture propagation conditions that enable intracellular signaling mediators to stabilize the molecular circuits and functions of pluripotency via transcriptional and epigenetic mechanisms inter alia. Understanding these sustained networks that auto-equilibrate in steady states of reciprocatively regulated pluripotency factors that globally organize the stemness genome, transcriptome, and proteome, is crucial for future strategies of regenerative medicine. All stem cells are regulated by such stemness and differentiation circuits and ESC pluripotency is still their best model system. A self-sustaining network of transcriptional activators and repressors specifies the undifferentiated identity and gatekeeps differentiation via hierarchical master regulators like Oct-3/4, Nanog, Sox-2, and Klf- 4 at its core. These factors are of the highest functional-regulatory order and can even globally reprogram specialized body cells into iPSCs. The mechanisms of stemness and differentiation are key to decode the postgenomic program of life and its overall complexity represents a new organizational challenge for system biology and open science, which will help to generate cell-based replacement materials and to heal genetic diseases like cancer. As a proof-of-principle, this work further analyzes the role of the signaling mediator $\beta$-catenin in stemness and pluripotency and reveals new transcriptional modalities, which further exemplifies the requirement to dissect all multi-functionalities in an open systems biology approach of open science and innovation, and proposes a stem cell hub termed iStemCore.

The Role of $\beta$-catenin in Embryonic Stem Cell Maintenance Circuits and iPSCs -

An International Systems Biology Approach of Open Science and Innovation

J Embryol Stem Cell Res 


\section{Journal of Embryology \& Stem Cell Research}

Keywords: ESCs; IPSCs; Oct3/4; Nanog; KLF-4; Sox; Stemness; Beta-Catenin; CTNNB1; Cadherin; Stem Cells; Maintenance; Lif; Pluripotency; Wnt; Signaling; Systems Biology; Biomarker; Open Science; Cdh1; E-Cadherin; Cell Adhesion; Rex-1; TERT; Transcription; Factor; Network; Reprogramming; Biomedicine; Regenerative Medicine; Tissue, Cell; Engineering; Organ Replacement; Cell Fate; Cell Identity; Circuits

Abbreviations: ESCs: Embryonic Stem Cells; IPSCs: Induced Pluripotent Stem Cell; HTS: High-Throughput Screening; HSCs : Hematopoietic Stem Cell; LOF: Loss-OfFunction; GOF: Gain-Of-Function; TERT: Telomerase reverse transcriptase; CTNNB1: gene encoding the $\beta$ catenin protein; Rex-1: Reduced expression 1 protein of the ZFP42 gene; Cdh-1: gene encoding the E-cadherin protein; iPSCs: induced pluripotent stem cells; SOPs: standard operating procedures; GSK3: glycogen synthase kinase

\section{Introduction}

Embryonic stem cells (ESCs) and induced pluripotent stem cell (iPSCs) are powerful cellular sources of regenerative medicine for future biomedical replacement strategies [1]. Systematic research of ESCs and iPSCs can better our understanding of cell fate and identity, and the intrinsic molecular programs of signaling and transcription [2] that form the basis of rational stem cell engineering, stem cell engineering. Propagation of ESCs or ESC-like iPSCs requires the in vitro maintenance of their pluripotent state, which is achieved by specific culture conditions and protocols that stabilize the network of molecular stemness factors via activated or repressed signaling cascades that promote circuits of intracellular transcriptional regulators $[3,4]$. ESCs and iPSCs are relatively symmetrically dividing adherent cells that grow in colonies and form cell-to-cell adhesion via homotypic E-cadherin binding, which results in an especially tight colony formation on a supporting, mitotically inactivated, fibroblastic feeder layer. $\beta$-catenin is a protein that connects the intracellular tail of the membrane Ecadherin adhesion complex with $\alpha$-catenin that links it to the cytoskeleton and provides physical stability and cell and tissue architectural properties. A signaling role of cell adhesion is less clear and is still controversially discussed, as its signaling role in the cytoskeleton, where it provides physical stability and cell and tissue architectural properties.

ESCs and iPSCs can also grow and attach in vitro on extracellular matrix proteins, collagens in gelatine, e.g. via integrin's that also enable motility and a swarm-like density-controlled cell migration. Gelatine allows their growth without feeder layer cells on a more chemicallydefined and more static ECM matrix where they grow in a less heightened colony-type colony-type and more in a migratory and flattened morphology due to the predominant feeder-free matrix-cell interactions and still maintain their ground-state of stemness including all major and recent stemness markers [5]. ESCs and iPSCs, which are derived from body cells reprogrammed into ESCs [6], have to be split regularly, every 2-3 days, due to their cell cycle speed and division pattern, or they would overgrow in colonies and start to (differentiate) in an uncontrolled way, once a colony becomes spherical or multilayered. They are usually and very widely cultivated in serum-containing culture media with the standard tissue culture ingredients but supplemented with Lif (leukemia inhibitory factor), or alternatively with $2 \mathrm{i}$ that is a mixture of Lif and inhibitors that co-inhibit MEK and GSK3 [7] in the case of mouse ESCs. 2i, which is an optional cultivation method [7] that makes use of kinase inhibitors to further ensure the ground state also via epigenetic mechanisms of global genomic DNA hypomethylation inter alia (i.a.). Although differences between mouse and human ESCs and iPSC exist, including variations in the culture protocols, SOPs, and molecular mechanisms, they are still highly comparable. It is more feasible to study mouse ESCs and iPSCs, which are more tractable in embryology and better understood [1], while human iPSCs are more relevant for preclinical and biomedical research, and can serve as a medical model system for drug discovery or molecular medicine.

Oct-3/4, Nanog, and Sox-2, i.a., are the masterregulators of the pluripotent embryonic stem cell state that form the genomic and transcriptional basis of continuous self-renewal and propagation of the dividing ESCs or iPSCs [1,3-6]. Today, they are generally described as highly hierarchical and powerful cellular regulators that act via genomic, epigenetic, transcriptional and regulatory molecular switches and gatekeepers that act in concerted action to preserve the capacity of ESCs and iPSCs to differentiate into all somatic and germ line cells of the body [1,3-7]. There are many further prerequisites of the pluripotent state and capacity like many additional core and peripheral factors, cell physiology and cellular 


\section{Journal of Embryology \& Stem Cell Research}

contexts. However, the pluripotent characteristic is still best and only fulfilled in the implanting and developing blastocyst and not fully in vitro where only partial differentiation routs are accomplished [1]. ESC, iPSC or ICM differentiation that can yield a fully functional body, all organs, or replacement parts, is still only achievable in the blastocysts and in vivo [1], which is unethical and not allowed. Nevertheless, in vitro differentiation protocols of today using ESCs and iPSCs yield many more functional cell types of the body than decades earlier and they are still improving. Furthermore, it has recently become possible to even mimic implantation of the early embryo in vitro [1]. Although such in vitro cultures still require a blastocyst as the perfect developing vehicle for the ESCs or iPSCs [1], such blastocysts can be also engineered in vitro by surrounding ESCs or iPSCs with artificial or natural trophectodermal cells [1]. Most of the in vitro differentiation protocols do not make use of a blastocyst, as this is technically more difficult, and the methodological dream is in vitro, although this is not feasible for many tissues due to the regulative cylinder stage of gastrulation and primitive streak pattern formation [5], which is impossible to fully resemble without further guidance. Later stages of differentiation can be used for regeneration like adult stem cells and regenerative cells and tissues, or maybe also using natural and artificial materials like structural ECM matrix grids or organ parts that can be generated in vitro with cells and various re-assemblages. The blastocyst seems important at the very beginning and might be essential [1], as the blastocyst is the natural surrounding structure from which ESCs were and are initially derived, i.e. from the inner cell mass, abbreviated as ICM [1]. iPSCs and ESCs resemble the ICM state including all of the ICM features and pluripotency factor circuits to be studied [3-6].

These ICM, ESC and iPSC features of pluripotency and stemness are functionally preserved on the molecular level in the nucleus and in the cell by a steadily required transcription factor circuit of core stemness master regulators comprising Oct-3/4, Nanog and Sox-2, i.a., that can be further extended by additional factors and peripheral factors and their mechanisms [3-5], which they are also linked to identity, fate, and also some housekeeping and cascade-regulatory sub-functions and sub-targets. In sum, the core and peripheral stemness circuits bear some key transcriptional and epigenetic mechanisms that exert direct global control over many thousands of target genes, directly and indirectly, via cascades of transcriptional regulation that can be either repressive or activating [3-5]. The underlying transcriptional logic is to be better understood. It comprises promoter blueprints and transcription-factor wiring schemes for ESCs [3-5] and iPSCs [6], which is a stable stemness activation code of core factors that allows the propagation, pluripotency, and reprogramming. This meta-stable stemness state is much more stable than any developmental transitioning states but at the same time it is also much less stable than any terminally differentiated cell type - it is a ready-to-differentiate state for all directions and germ layers, awaiting the right cues and triggers that can be natural or artificial in vitro or in vivo, one might categorize. How many artificial states and efficient differentiation routs might exist is not clear by today. They are researched with the goal to generate cells of the next meta-stable and propagative stemness state, i.e. precursor and tissue stem cells and adult stem cells [26]. It seems likely that additional meta-stable adult stem cell states exist that can be exploited once they are better and further explored: for example primed ESCs, activated ESCs, epiblast ESCs, tissue originator stem cells, precursors, adult stem cells, and more - they are like the next milestones.

An open science [8] systems biology understanding of ESCs and iPSCs [6,9] can hold the key and medicinal promise of regenerative medicine for many human diseases and for molecular medicine. Still, the right open science approach for systems biology is missing for all scientists [9] like an international free platform and hub as systems biology can only be a collaborative IT- and biobanking service for all of the laboratory scientists and all of the theory contributions. Patient-specific iPSCs can be used to develop better specific and rational assays for high-throughput screening, HTS, and HCA, in drug discovery and $R \& D$, and for new methods to research and treat human diseases [6]. Developing a platform and a better understanding of the highly combinatorial signaling and transcriptional cues, cell contexts and responsiveness [2] is still needed for a systemic breakthrough. ESCs and iPSCs are an ideal tractable genetic-cellular system and agile tissue model to study early differentiation and stemness. These mechanisms must be better explored in order to understand later stages of development and disease that are also regulated by such core circuits. Understanding the molecular networks, programs and circuit mechanisms of cellular, tissue and organ blueprints are the next quantum leap for rational targeted stem cell medicines and systems platform is needed.

In the last decades, international networks of outstanding stem cell scientists have elucidated the stemness transcription factor core circuit and many of its 


\section{Journal of Embryology \& Stem Cell Research}

components including the many direct and indirect targets and cascades and systems of transcriptional logic also using the system tools of omics and bioinformatics $[2-6,9]$. However, all efforts and endeavors of this new life science technology still needs a systems biology platform that is accessible for all scientists in reading, writing, bio banking, materials, incentives, models, data, publications, bioinformatics, databases, omics data and transparency via open science [8]. Decades of previous stem cell research has already carved out many interaction and promoter maps and key transcriptional logics [1-6,9] that always sees Oct-3/4, Nanog, and Sox-2 and additional factors like Klf- 4 at the very heart of a core master regulatory transcription factor network that costabilizes each factor to reach the metastable stem cell state $[1-6,9]$ and many more outstanding contributions. Stem cell maintenance requires extrinsic signaling modulators of important signal transduction pathways that play a fundamental role in development, disease and biomedicine. Several additional signaling factors, cofactors, second messenger, branches, and components now also extend this ESC and iPSC core transcription factor network with pivotal roles in the early stemness and differentiation states. They can deliver further specificity and direction and trigger metastable statetransitions while reaching out to additional targets and often serve as co-activators on promoter MPCs (multiprotein complexes). The big prevailing central dogma is that signaling drives transcription that directs stem cells towards differentiation or stemness. The linearity of this dogma is questioned here, as factor multifunctionality, cell adhesion, and circuit network effects could be also in play, which leads to circuit, network, and amplifier, transitors, and deflector effects.

Signaling pathways comprise, Jak/Stat, Wnt/ $\beta$-catenin and non-canonical non- $\beta$-catenin Wnt signaling (PCP), TGF $\beta / B M P, T G F \alpha$ or EGF, Notch, Hedgehog (SHH, IHH, DHH), MAPK and ERK, cyclic nucleotide pathways (cGMP, cAMP), IP3/DAG, redox, calcium, NO, lipids, cytokines, growth factors, peptides, neurotransmitter, RTKs pathways (FGF, EGF, VEGF, PDGF, GDNF, and many more), GPCRs, ECM (integrins/CDs), death signaling (TNF $\alpha$ ) [10], Hippo, ECM, minerals, osmosis, heat, $\mathrm{pH}$, metabolism and metabolites, basically also many organic and inorganic small compounds, and even more. Typical signaling is usually activated by a first messenger, a cytokine, ligand or growth factor and the signal is subsequently amplified and relayed intracellularly by second messengers that trigger the cellular response such as transcriptional activation. The interpretation of all signals, also known as biosemiotics, is accomplished by the entire cell as a responder, and activation of transcription plays a fundamental role in its molecular responsiveness to signaling. Hence, anthropocentric interpretations must always include the context that can read the signaling and that can write the signal. These signaling factors are known modulators of promoter-associating MPCs that can modify the chromatin structure and regulate and activate transcription in development and disease and are of highest biomedical relevance and triggers of key cellular states and functions. Second messenger and secondmessenger-like signaling molecules, and the activated transcriptional modifiers can interact with MPCs of many major classes of transcription factor families. They can also directly interact with several core stemness circuit factors like Oct3/4, Nanog, or Sox-2, which explains the crucial link between signaling ligands, transcriptional patterns, and cellular fate. Signaling ligands or molecules (inhibitors) in stem cell media are used to support this global transcriptional stemness state and self-renewal of ESC or iPSCs [3-6].

Growth factors in FBS and addition of Lif (leukemia inhibitory factor) or kinase inhibitors in $2 \mathrm{i}$ media [7] are regularly used today to activate signaling, epigenetics and transcription to ensure the stability of the core master regulators and the global transcriptional regulation of the iPSC and ESC genome in regenerative medicine [3-7]. Oct$3 / 4$ and Sox-2 often act in tandem and in concert via DNAbinding proximity on promoters to activate or repress target genes [3-5]. Nanog protein levels might be the key triggers that regulate the undifferentiated meta-stable pluripotent state [11], maybe as one of the several internal switches of differentiation of the early stemness states starting from the ground state to the epiblast state [5] but the regulation is likely not simple but multifactorial or timely concerted. Furthermore, monoallelic regulation of Nanog is one of the key stemness mechanism in Lif-containing media [12], while $2 \mathrm{i}$ inhibitors act in two ways epigenetically [7] to activate the second allele of Nanog that in turn potentiates stemness factor gene transcription also via a more permissive chromatin structure [11,12], which might naturally resemble the native blastocyst setting [1]. ESCs and iPSCs of the mouse are a very important model system in which Lif and 2i FBS media is used [7]. These stimuli maintain the meta-stable state via activated components of signaling transduction pathways, which are also known as pathway nodes. Lif phosphorylates and activates the intracellular second messenger Stat-3, while FBS can back-level activate many signaling pathways like TGFs and RTK signaling. Wnt represses GSK3 that phosphorylates $\beta$-catenin and targets it for ubiquitination 


\section{Journal of Embryology \& Stem Cell Research}

and proteasomal degradation $[5,13]$. Activated $\beta$-catenin has various differing combinatorial patterns of posttranslational modifications. If its destruction box on exon3 is not phosphorylated it cannot suitably bind $\beta$-TRCP and accumulates by escaping default degradation of the proteasome [13]. Many more protein-protein interactions and molecular mechanisms in Wnt signaling play pivot roles in development and disease $[13,14]$. Wnt signaling, and signaling in general can pattern the organismic blueprint and can mediate tissue homeostasis. Ectopic activated Wnt signaling makes and can duplicate the first anterior-posterior body axis, while BMP2/4 has a role in bilateral symmetry and the dorsoventral axis $[2,13,14]$. This patterning and differentiation cues make signaling so interesting for stem cell research $[2,5,13,14]$. While $\beta$ catenin's role with cadherins in cell adhesion and TCFs in transcription is generally understood, the mechanisms of additional interaction factors can still be further elucidated. Also, many additional functions on RNA, DNA, ECM, and proteins are thinkable. Dissecting and exploring the fundamental roles of $\beta$-catenin and signaling components in general is still very important and required to understand the cruicial mechanisms. Fundamental roles in ESCs and iPSCs are still to be better, e.g., how can the positional information, like the axis orientation, given by signaling and cell contexts, be used to channel the differentiation of cells and tissues for a significant medical demand and supply.

$\beta$-catenin has a role in stemness and differentiation of ESCs [2] and iPSCs and elicits these differing functions via its interaction factors. Traditionally viewed and simplified, $\beta$-catenin has two fundamental roles in the cell: (i) as a virtual second messenger of Wnt signaling that translocates to the nucleus to transcriptionally activate target genes via TCF/Lef-1 transcription factors that can modify epigenetic promoter states [13-15] and (ii) in homophilic cell-to-cell adhesion, by connecting the cytoplasmic tail of transmembrane E-cadherin adhesion complex between two cells with the cytoskeleton, and (iii) non-TCF-dependent ways [2,15]. It could be recently shown that $\beta$-catenin can also bind to non-TCF transcription factors, and to the stemness core factors like Oct-3/4 and Nanog in ESCs [5]. Several lines of evidence suggest that this binding plays a crucial role in ESCs and iPSCs: first, ESCs in the ground state do not show any transcriptional activity of $\beta$-catenin via TCFs, while a lack of $\beta$-catenin destabilizes the ground state of ESCs as shown by a loss of marker genes like Rex-1 [5]. Oct-3/4 and Nanog repress $\beta$-catenin TCF transcription in ESCs and other cells very likely due to this binding [5], IP and WB experiments were also done (not shown).
Subsequently, we could show that TERT transcription is regulated by $\beta$-catenin in a TCF-independent way and that Klf-4 and epigenetic mechanisms are in play [15]. TERT is an enzyme that extends cellular lifespan by prolonging telomere repeats on chromosomal ends and chromosomal ends via the RNA primer component TERC (Telomerase RNA component). Telomere and biomarker stability drives stem and cancer cell viability and many additional functions of highest relevance for the dateless cell.

This review and research provides a general overview and presents a new stem cell marker panel that further helps to understand the dissected role of $\beta$-catenin in ESCs and iPSCs. It suggests a systems biology approach and gives a proof-of-principal, proof-of-feasibility, and proof-of-way-to-go when it amends our understanding of signaling co-factors, here $\beta$-catenin, on the transcriptional stability and integrity of the core transcription factor circuit and its peripheral network and mechanisms of the pluripotent stem cell state of ESCs and iPSCs, with a longstanding impact on systematic regenerative medicine.

\section{Methods}

ESC lines and tissue culture procedures and methods: wild type mouse ESCs, heterozygous $\beta$-catenin ${ }^{\text {flox/-, }}$ homozygous double knock-out LOF (loss of function) mutant $\beta$-catenin-/-, heterozygous GOF (gain of function, and a stabilized isoform with hyper-activated functions) $\beta$-catenindEx3/wt [16-18] were analyzed. ESCs were maintained in an undifferentiated state in 15\% FCS containing DMEM medium supplemented with nonessential amino acids, L-glutamine, $\beta$-mercaptoethanol, and self-made LIF and routinely propagated on MEF (mouse embryonic fibroblast) coated-culture dishes or on $0.2 \%$ gelatine-coated dishes for homogenous profiling or validation experiments. To assure the highest level of reproducibility, identical cell density and culture conditions were assured. The RT-PCR experiments were not published initially and were the basis of the subsequent TERT project [15] RT-PCR experiments were carried out using standard procedures and procedures and primers were designed and their functions were validated based on the respective PCR product fragment length, see Figure 4. A new RT-PCR primer combination was used for telomeric repeats that yield a nested product ladder of the repeating sequences using standard PCR procedures. The remaining ESCs and RT-PCR procedures were performed as previously described in Anton [2].

System Biology Approach for ESC and iPSC Stemness and Differentiation (method infrastructure hub 


\section{Journal of Embryology \& Stem Cell Research}

proposition). This Open Science and Open Innovation $[8,19]$ core facility will be a free and open service for the entire world of stem cell sciences once the international funding becomes available for the proposed international research project (a hub method for the field). Patientcustomized stem cells will be part of the Biobank and the Systems Biology Database and platform for models, publications, scientific discussions including reporting and all related information. Researching corporations and academic institutions will not have to preserve all lines in a redundant way, which will save costs, no cell lines will be lost, and this new and global platform also enables and assures that all information will be orderly stored, characterized and openly discussed and continued. Access and plain level contribution for postdoctoral research professionals, Ph.D., next to PIs is another core feature of iStemCore, the International Stem Cell Research Core hub, and Open Science $[8,19]$. It will also shift the field into a new way of publishing. Instead of stories, individual new findings and data could be added to models or new models created, according to the scientific method, which will be quality managed in an SM-QMS (scientific method quality management system), accessible for all. Also, iPSC characterization and genomic repair, e.g. via CRISPR editing, can be systematically studied. Full central information and resources for decentral but systematic research would also pave the way for all other sciences.

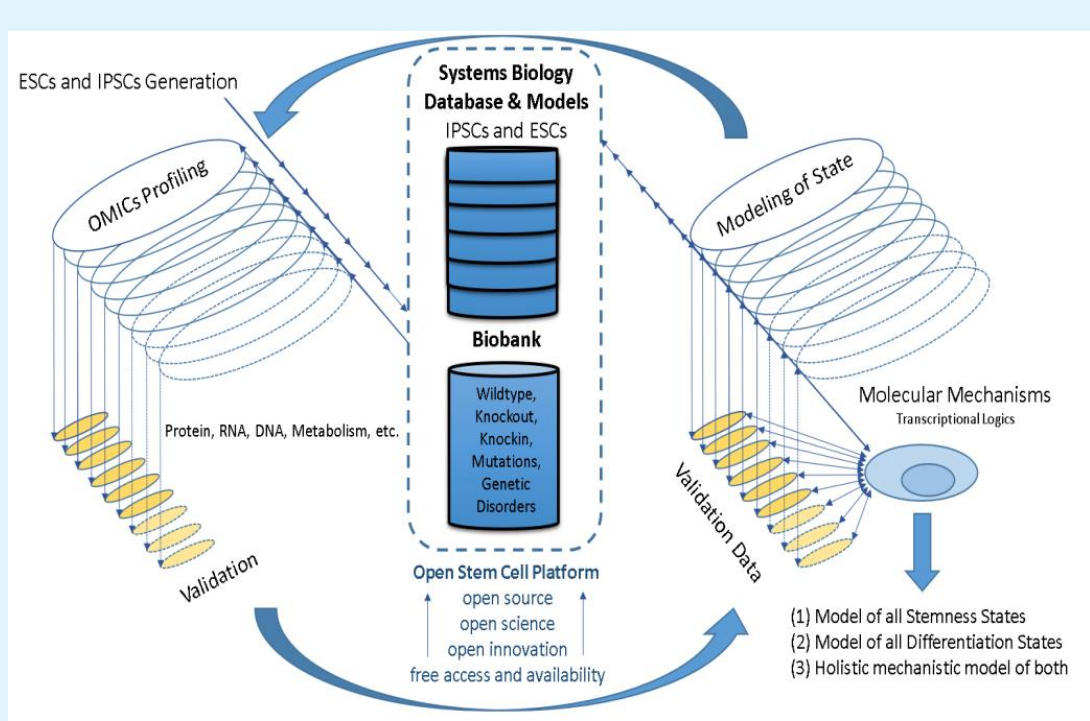

Figure 1: System Biology Approach for ESC and iPSC Stemness and Differentiation. This Open Science and Open Innovation (David 2004, Chesbrough 2003) core facility will be a free and open service for the entire world of stem cell sciences once the international funding becomes available for this international research project. Patientcustomized stem cells will be part of the Biobank and the Systems Biology Database and platform for models, publications, scientific discussions including reporting and all related information. Researching corporations and academic institutions will not have to preserve all lines in a redundant way, which will save costs, no cell lines will be lost, and this new and global platform also enables and assures that all information will be orderly stored, characterized and openly discussed and continued. Access and plain level contribution for postdocs, Ph.D., next to PIs is another core feature of iStemCore, the International Stem Cell Research Core hub, and Open Science (David 2004, Chesbrough 2003). It will also shift the field into a new way of publishing. Instead of stories, individual new findings and data could be added to models or new models created. Also, iPSC characterization and genomic repair, e.g., via CRISPR editing can be systematically studied. Full central information and resources for decentral but systematic research would also pave the way for all other sciences, and the scientific method would be again in place, even SMQMS assured.

\section{Results}

To reveal the transcriptional logic of the circuits in the stemness transcription factor network that sustain pluripotency, I have early on proposed a global systems biology approach as an open science hub for ESCs and iPSCs, mouse, human and additional species, around 2004 and 2008, also for iPSCs via fair platform collaboration, 


\section{Journal of Embryology \& Stem Cell Research}

and as a crowdsourcing-based free and open science hub with a central database and bio-bank service and reading and writing rights for all scientists (see Figure 1). By modeling the boolean logic of stemness and differentiation [5], not shown due to PI barriers to publication], several intermediate transition states of ESCs and iPSCs became apparent also with regard to marker and master regulator expression, mechanism, and function. Already a few biomarkers gave rise to a higher level of complexity in the Boolean models that also make comprehensive and somehow standardized data and databases an important pre-requisite. Obviously, global systems biology approaches [3, 9] have already become a necessity and now also open science and open innovation $[8,19][8,19]$ establishing a fairer, free contributive and accessible, and better functioning world of science that is inclusive for all researchers. A collaborative platform platform (see Figure 1) with suitable legal and symmetrical information and symmetrical IT agreements, free MTAs, free data, centralized bio-banking and data storage, that collects and distributes information and materials in a fair, level playing field, and transparent way, for $\mathrm{PhDs}$ and postdoctoral professionals, not only for faculty and PIs: an international hub that would boost stem cell research by delivering the missing centralized platforms, in short: the iStemCore.

Demarcations of this hub of regenerative medicine and stem cells could, would, or shall be embryonic stem cells of mouse, mammals, and men but should include all generated iPSCs, e.g. men but including all iPSCs, e.g. patient-specific iPSCs [6]. Additional hubs could be generated for all remaining stem cells, all types of adult stem cell (HSCS, MSCs), and a hub for cancer and hubs for all human diseases that make all materials, methods, theories, and information available to all.. The hub would be also defined via open science and open innovation as fair platforms of collaboration and participation are needed, internationally, like better career paths and fair chances and enough jobs for scientist.

With regard to systems biology [3, 9] and open science and R\&D innovation $[8,19]$ of embryonic stem cells and iPSCs [6], a more centralized hub (see Figure 1) can ideally amend and connect the decentralized researching groups, institutes, companies and investigators, for example in omics, data and material sharing, universal MTAs, modeling, bio-banking, and in all free access, open and affordable material transfer questions using one simple MTA for all and a fast on-demand shipping, with the idea in mind to also, save costs by avoiding redundant bio-banking or the need to re-research, to reinvent or to recreate iPSCs or ESCs, and all alike. As previously mentioned, ESCs and iPSCs are an ideal model system for the specific biomedical question and basic research to develop new procedures and findings for biomedical question, as (i) they can be patient cells with all genomic and physiologic traits, (ii) could provide the ideal test setting once protocols become available for differentiated cells, (iii) to study molecular medicine and gene editing strategies in an immortal model system of standardized patient cell (iPSCs), (iv) to screen, and study molecular mechanisms (physiological genes can be active or activated in ESCs). Homogenously cultivated ESCs or iPSCs are a suitable model system to perform tissuespecific transcription and protein profiling and omics approaches due to the high purity of the ICM-tissuederived stem cell type: e.g. most tissues or are even more heterogeneous than ESCs, which are thus the better model system of stem cells. ESCs can also exist in interchanging heterogeneous sub-states but this ICMtissue state is still much more homogenous than most tissues or purified stem cells, like mesenchymal (MSCs), hematopoietic stem cells (HSCs), or even cancer stem cell (CSCs) and their niches or perhaps all tissue biopsies. ESCs and iPSCs are thus the ideal model system for biomedicine.

This experimental presetting also enables the highly reproducible and highly standardized comparison of transcription network profiles of a very interesting pluripotent tissue state. By comparing many subsets of ESC and iPSC lines that carry loss-of-function (LOF) and, or, a gain-of-function (GOF) mutations, or important biomedical crucial genetic variations, a better understanding for regenerative medicines and cellular therapies can be developed. Furthermore, such ESCs and iPSCs can be screen and analyzed for important and supportive core factors of the stemness and differentiation networks by comparing the profiles and mechanisms to the wild-type ground state of stemness and many further strategies. Furthermore, an iStemCore (see Figure 1) could make it possible to develop more medical read-outs for therapeutics and diagnostics based on iPSCs in the stemness or in vitro differentiated states [6]. The cell lines that were created would be properly stored and distributed and systematically analyzed Figure 1. Different methods and laboratories could collaborate and compete to find the best understanding and breakthroughs on a fair and informative open access platform of open science, which should be transparent, collaborative and accessible $[8,19]$. This system biology project could have free access bio banks and free access databases and information for ESCs and iPSCs that are 


\section{Journal of Embryology \& Stem Cell Research}

accessible for all researchers internationally, free for research purpose mainly, and affordable for the industry, which could save many redundant costs and resources for all (see Figure 1). It would help to develop biomedical read-out cellular assays using the iPSCs cell lines that carry an important biomedical mutation of a human disease. All scientists would have access to the latest research and research materials that would make international stem cell research one fair and more competitive collaboration with better incentives for researchers that would also require sustainable career paths (sustainable career path for scientists in academia and the industry are highly needed, as this top-level workforce is still disadvantaged worldwide and fairness remains highly elusive). The molecular mechanisms and logics $[3,4,9]$ can be best revealed if all facts are systematically brought together to connect the dots in open science's systems biology free platform for all.

There are and will be (some) major exemplary challenges or biases of this systems biology approach (Figure 1): (i) very important master regulators of the stemness state are so important for the viability, isolation, and cultivation of ESCs or iPSCs. Due to such various reasons, some ESCs with gene mutations cannot be cultivated as a KO (LOF) would destabilize the stemness state that fully requires the factors to maintain the transcriptional integrity of the core pluripotency network and, or, the viability and proliferation of the cells. The network would immediately destabilize and the cell cannot be cultivated. As ESCs are isolated from blastocysts many mutations might have escaped the findings when only a lack of offspring was diagnosed and early stages were not further analyzed. (ii) How to assure the scientific method in international team play of research - a general question that has not been resolved and which halts science worldwide and hinders any progress in our understanding. The SM-QMS approach proposed for the iStemCore could help to find a way how the scientific method can be implemented internationally.

As with most of the real world and realistic systems biology approaches, one cannot implement them alone in your home institution and faculty, or in your institution, because they are international mega projects. Hence, systems biology is on the road to becoming international projects per se and always and almost, and less a national, or of one institute. Maybe it will require international funding and an international open science and open innovation $[8,19]$ infrastructure to function Figure 1.

As a proof of principle, to test if such systems biology approaches would work in general and in the in-depth details, one can have a look at suitable ESC research projects. This simple example takes mouse ESCs that are mutant for an important signaling component that can be characterized, analyzed and compared with respect to pluripotency network perturbance [2]. These datasets can be compared to others in a second step, for example, once iStemCore is established (Figure 1). Today's comparisons are maybe not standardized enough in many cases and standardized validation is also missing on the international systems biology scale (See Figure 1). This comparison of $\beta$-catenin LOF ESCs was subsequently further extended to dissect the function of $\beta$-catenin for a more systematic systems biology low-budget proof-ofprincipal approach (data not shown). Signaling components play a fundamental role in the transcriptional activation and co-activation, repression, and co-repression of iPSC and ESC master regulatory pluripotency transcriptional network. They can pull the trigger for stem cell maintenance and in vitro differentiation by stabilizing or destabilizing the manifold and feed-forward transcriptional stability of 0ct-3/4, Nanog, Sox-2, Klfs and additional important core factors that regulate the core and peripheral network $[2,3,4]$ and global transcriptional programs, pluripotency and all of the cellular states and early fates, transcriptionally and epigenetically via signaling, which is still today's view. Lif/Jak/Stat3 maintains mouse ESCs in FBS and the contribution of additional pathways is of interest. The Wnt/ $\beta$-catenin signaling pathway can serve as an example.

Wnt/ $\beta$-catenin is one of the most important signal transduction cascades in the animal kingdom and plays fundamental roles in patterning, development and human disease [14]. To further dissect the basic molecular mechanism of $\beta$-catenin in (a) signaling/transcriptionalactivation versus (b) cell adhesion, the following ESC lines were tested, profiled [2] and further characterized: (1) $\beta$ catenin LOF (double knock-out), (2) $\beta$-catenin GOF (deltaexon3/wild-type, which is an artificially hyperactive stabilized isoform that escapes proteasomal degradation due to the lack of the destruction box in exon 3), (3) Ecadherin LOF (double knock-out, lacking the major cell adhesion mediator in ESCs and iPSCs), (4) heterozygous $\beta$-catenin mutants (a "recessive-type" of mutation as the second allele compensates for the loss), and (5) wild-type ESC lines that were published before (see methods) but were not subjected to a system biology characterization with the goals mentioned in Figure 1 . According to the prevailing theory in 2004-2008, (a) $\beta$-catenin would have a functional role with TCFs in transcription, and (b) a physiological role on cell adhesion via E-cadherin, and (c) non-TCF-dependent transcription of $\beta$-catenin [2]. Also, E- 


\section{Journal of Embryology \& Stem Cell Research}

cadherin, and its switch in early differentiation or in metastatic cancer to EMT [20] or to N-cadherin, i.a., seems to play a role in the very early differentiation of trophectoderm - before ESCs or ICM has finally formed and in tissue formation $[16,17]$. Still, the role of 1-5 and a$c$ in ESCs and iPSCs was and is still not fully resolved and this work and a systems open science, as proposed in Figure 1 for the iStemCore, can be the systems biology solution $[8,19]$.

How does the experimental perturbance of one molecular component affect stemness, pluripotency, and physiological functions, can now be better answered in the iPSCs and ESC system by developing new standardized assays and readouts for all equally treated available cell lines, comprising omics and target validation (Figure 1). Based on the underlying 'molecular mechanisms', blueprints could be drawn in a more comparative way to answer some key questions and research objectives via iStemCore Figure 1 . To stay with the concept's example, how does a loss of a key signaling component, $\beta$-catenin, affect the molecular integrity and mechanistic circuit transcriptional homeostasis? A total loss of $\beta$-catenin destabilizes the transcriptional integrity of specific parts of the stemness network. Several markers are highly reproducible and highly significantly altered in many independent experiments and projects [5]. $\beta$ - catenin knockout (KO -/-) ESCs from two independent laboratories have both yielded exactly the same effect on the molecular biomarker phenotype $[5,15]$. Subsequent assessments have also shown this and the muchdiminished cell adhesion effect. However, a discrepant work exists that should be mentioned, by Lyashenko 2011 [21] that does not see a cell adhesion or marker effect, which raises important methodological questions of systems biology that frequently arise in many fields. Systems biology can best report all discrepant works on one information and publishing IT platform that can help to solve these questions in the technical details and there are likely many hundred thousands of contradicting cases in the scientific literature if not even many more; platforms like iStemCore are the solution to better study all of the inconsitencies that may and that naturally can arise (see Figure 1), which shows the need of system biology, open science and future hubs like the iStemCore. Maybe the protein level or a different molecular weight isoform [21] were not entirely depleted for a sufficiently prolonged culture period that could yield a contrary phenotype in the contradicting method. Clearly, the loss of ESCs stemness markers in $\beta$-catenin KO ESCs has been highly reproducible in different labs, as shown by the two independent $\beta$-catenin KO LOF ESCs that originate from two different and world-leading $\beta$-catenin laboratories [5] and this is newly also shown in Figure 2.

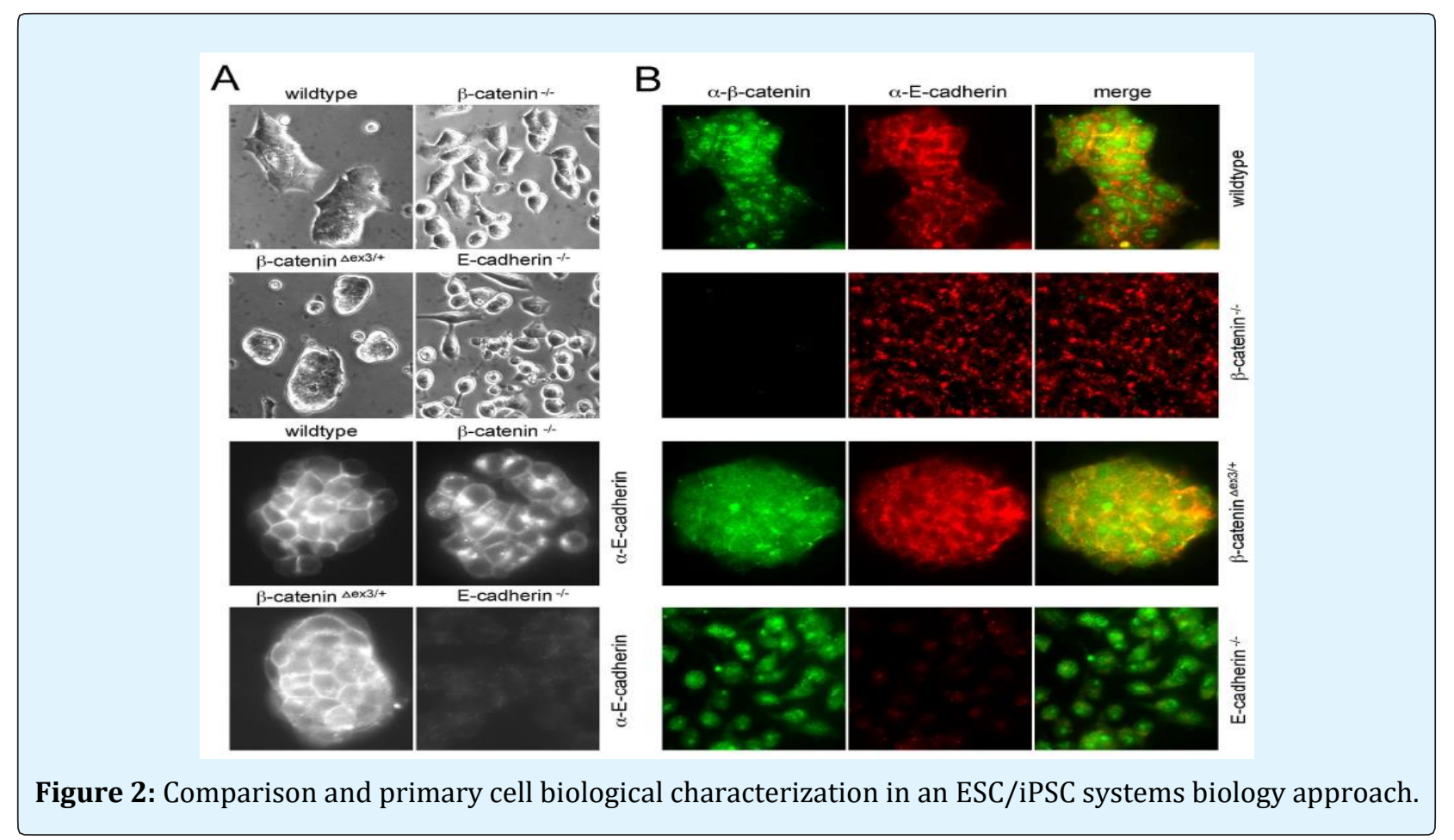

Roman Anton. The Role of $\beta$-catenin in Embryonic Stem Cell Maintenance Circuits and iPSCs - An International Systems Biology Approach of Open Science and Innovation. J Embryol Stem Cell Res 2018, 2(2): 000111. 


\section{Journal of Embryology \& Stem Cell Research}

Cell adhesion defects are seen in E-cadherin and $\beta$ catenin LOF (-/-) ESCs, while $\beta$-catenin GOF ESCs shown a more compacted and tightened colony morphology. The antibody is abbreviated as $\alpha$ in this figure. In wild-type cells, $\beta$-catenin is found at the membrane, cytosol, and nucleus (different antibodies reveal different levels of nuclear stain and nuclear accumulation, not shown). Ecadherin is mainly found at the membrane. In $\beta$-catenin KO cells it is lost and E-cadherin becomes diffusely distributed in the cell at a lower intensity and shows stain in endoplasmatic reticulum (ER) and intracellular inclusions. In ESCs with a GOF mutation of $\beta$-catenin (delta-exon-3/wt), there is a higher accumulation or density staining of $\beta$-catenin throughout the cell (cells are more compacted and heightened) and E-cadherin is increased in the cytoplasm and only very close to the cell membrane. A cell-to-cell adhesion happens much less and visually-morphologically weaker in KOs maybe via plakoglobin, as has been suggested elsewhere [2] and references therein). Please note the cytosolic accumulations of E-cadherin in inclusion bodies or ER if it cannot go to the membrane. All of the embryonic stem cell GOF and LOF lines are completely depleted of protein, as has been shown before in the literature and first descriptions $[16,17,18]$ and only show background staining of secondary antibodies at high exposure times, to see the signal to noise.

A highest level of comparability was also always assured in this proof-of-concept systems biology comparison of ESCs in two projects. Also, The conventional culture conditions have assured that differentiation was kept very low as measured by several bio-markers like 0ct-3/4 or TROMA-1 see Figure S1 [note: Supplementary Figures are included in the main text in this version].

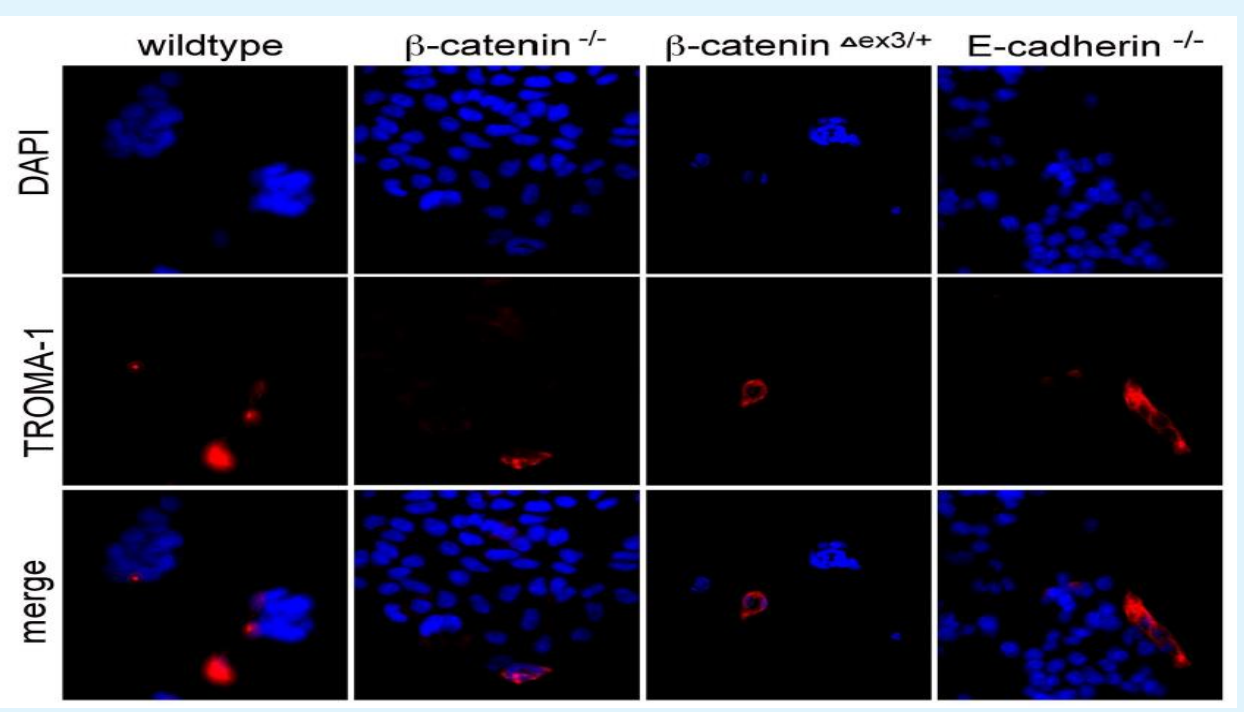

Figure S1: The level of differentiation is very low and comparable in mouse ESCs. A negligible level of only ca. 1\% can be seen in all culture. Future systems biology culture conditions may improve this standard further while making it available to all. A committee must decide on the suitable gold standards that meet the goals of comparability, utility, and feasibility. Wildtype, b-catenin, E-cadherin, and b-catenin-dEx3 cells all show very few Troma-1-positive cells that could be differentiating..

Representative examples of stained cells are shown, while $99 \%$ of ESCs are TROMA-negative. This highly homogenous culture and differentiation state allows a direct comparison of all ESCs lines tested. Please note that most tissues or adult stem cells have a lower homogeneity as compared to ESCs and it is more expensive and difficult to achieve such high numbers of cells that are needed for systems biology and the many omics approaches multiplied by all LOF and GOF cell lines: ESCs and IPSCs might be the most affordable stem cell system for omics and systems biology. Comparability of the cellular starting material is always of tremendous importance for all systems biology approaches, which is often unattended, and normalizations must be unbiased and can be optimized continuously. Normalization biases can often already become a challenge at this level, as 


\section{Journal of Embryology \& Stem Cell Research}

standardization on cell number, protein, RNA, mRNA, or marker level can all yield slightly different outcomes of the entire study and should be integrated or done in a most suitable way - and SM-QMS standards are needed.

$\beta$-catenin has a role in the transcriptional integrity of mouse ESCs [2]. Simultaneously, TCF-dependent transcription, as measured by Top flash reporter assays, is not activated in mouse ESCs, while a panel of key stemness marker genes is altered in the complete KO $\beta$ catenin ESCs [5, 15]. Hence, TCF-independent mechanisms are at work, as shown by Anton and Hoffmeyer [2, 15], and this work from 2008-2009. This could include more than one factor, Klf- 4 is one of them [15], but additional stemness factors could be also in play, as binding to Oct-3/4 or Nanog could further explain additional co-activator effects of $\beta$-catenin on the transcriptional regulation without TCFs. Furthermore, Oct-3/4 and Nanog can both represses Wnt/ $\beta$ catenin/TCF-activity in ESCs and other cells [2]. Also, in human embryonic stem cells, $\beta$-catenin's role in differentiation is repressed by Oct-3/4 [22], which fits into the model of a modulator and trans-activator of transcription and in epigenetics $[2,15]$, maybe depending on the respective interaction factor of $\beta$-catenin: TCF might have a role in further triggering transcripts in differentiation and some in stemness and is repressed by Oct-3/4, Nanog, or maybe factors like Sp-5, while its role with Oct- $3 / 4$ and Klf- 4 is to promote stemness gene circuits and expression of important genes like TERT [5, 15]. This as the recent current working hypothesis and model can be verified, falsified, or further refined in more detail. This reveals that collaborative systems biology approaches $[8,19]$ are about to become crucial to making a future progress in all fields. These need resource platforms of fair and transparent participation for postdoctoral professionals, PhDs, and PIs, while bioinformatics is only the IT service part of it and a division of labor between IT and lab is also needed. Concomitantly, sustainable career paths for scientists are needed in the industry and academia, inlcuding all alternate entry, management and research routes.

A comparison of mouse ESCs that are homozygous or heterozygous for CTNNB1, the official gene name of the $\beta$ catenin protein, could show a dosage dependency in theory, but western blotting can reveal that the protein levels are very equal (Supplementary Figure S2 B). But the definition "recessive" must be maybe revised, as the question of a recessive phenotype can be assessed at different levels of detail: (1) signaling and adhesion is very similar and functions in the heterozygous mutant cells, but (2) there are some fine differences in some marker gene expressions that might be overlooked before. The fine-tuning of some stem cell biomarkers are slightly different in mouse ESCs that are mutant for one allele: see figure 3 and supplementary figure S2.

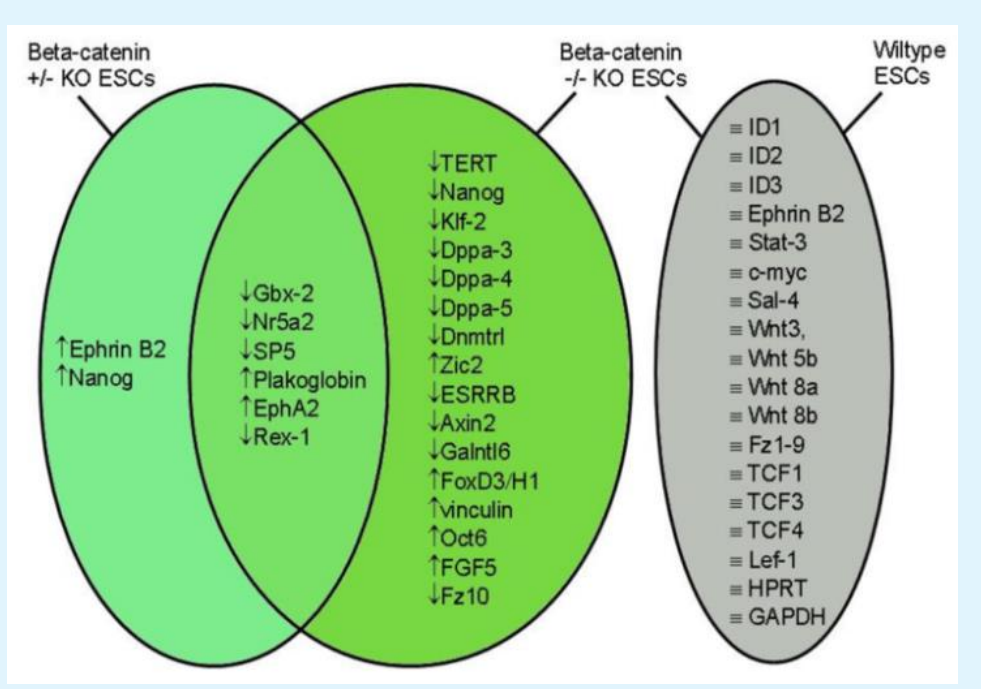

Figure 3: Dosage-effect of $\beta$-catenin in stemness transcription circuits of ESCs and iPSCs. There is a previously undescribed reproducible slight effect in heterozygous KO mutation of $\beta$-catenin in ESCs. 


\section{Journal of Embryology \& Stem Cell Research}

A

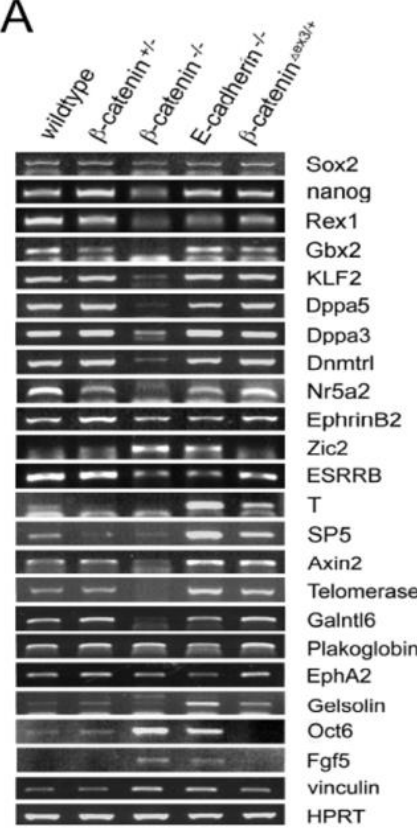

B

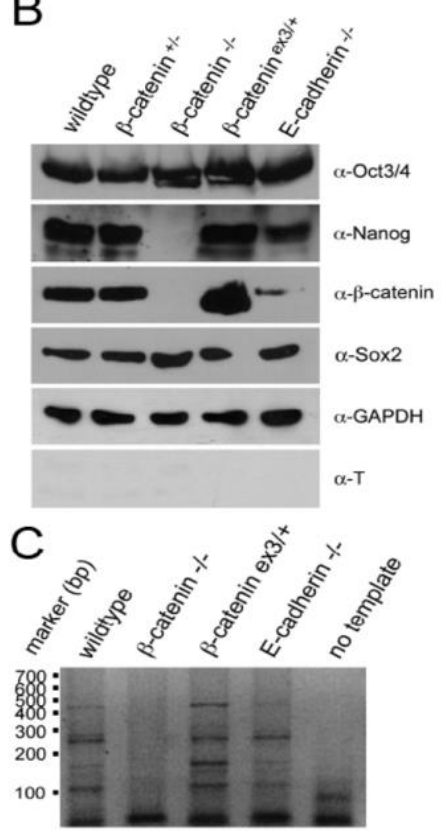

Figure S2: Stemness marker analysis using (A) RT-PCR and (B) Western Blotting (WB), and (C) a preliminary telomere repeat assay was established simply using affordable RT-PCR (2008-9). The result of shortened telomeres was fully verified later on with the suggested methods and protocols [15]. Comments of note for Figure S2: The protein-level of $\beta$-catenin is very much reduced in E-cadherin KO LOF cells (-/-) see and much increased in $\beta$-catenin GOF ESCs, see (B). Stemness biomarkers are regulated as shown in Figure 3 and Figure 5.

Hence, the LOF mutation is "not purely recessive in detail", although most functions and markers stay the same, there are some fine differences in the robustness of the level-precision of some marker genes (see Figure 3 and Supplementary Figure S2). As a result, it can be possible that specific genetics scenarios, like double heterogeneous knock-outs, can cause phenotypes in the mouse in a dosage-dependent fashion, which could be also a level-precision fashion. Theoretically, to arrive at the same amount of protein starting with only one allele and less mRNA, it seems to be possible that the protein stability is somehow increased in (+/-) $\beta$-catenin ESCs. This is very likely achieved by a mechanism that uses post-transcriptional modifications (PTMs) and might increase a PTM-isoform of $\beta$-catenin that might be a little bit more stable than in the wild-type ESCs. This different PTM-isoform of $\beta$-catenin could affect its transcriptional activity and marker gene expression in some cases.

Normally, the $\beta$-catenin protein level is adjusted by a protein degradation machinery involving GSK3, APC, Axin1/2, phosphorylation at $\beta$-catenin's exon 3 and subsequent ubiquity nation and destabilization via $\beta$ TRCP $[13,14]$ This leads to steady-state protein levels, while in exon- 3 mutants, the $\beta$-catenin GOF ESC lines; it causes dramatic accumulation of $\beta$-catenin protein and TCF-dependent transcription $[2,15]$, while in heterozygous $\beta$-catenin ESCs, the protein level is not changed or only very slightly reduced at almost undetectable $\beta$-levels. Western blotting shows no significant changes in $\beta$-catenin protein levels (Supplementary Figure S2), while the transcriptional level seems less. However, it is the protein level that is believed to exert the signaling, transcription, and cell adhesion function in ESCs and the organism. What if the mRNA or genomic level might already play a role, can be one of the basic questions to be asked in systems biology approaches, just to be complete with all tests and experiments and not to forget about a point; to be systematic in the analysis of all layer of regulation - and not only story hunting (this pilot work could only preliminarily propose such an analysis, project and the iStemCore). 


\section{Journal of Embryology \& Stem Cell Research}

Figure 2 shows several primers that were used in 2008 in the proof-of-principal experiment of systems biology via semi-quantitative RT-PCR experiments, that were highly reproducible and could be extended and followed up subsequently with alternative methods. Until today there is no international systems biology approach that has established the iStemCore as the funding and jobs are still missing: the main reason of this preliminarity.

A summary of the expression data is given in Figure 3. In the grey circle, all gene mRNA transcripts of the respective ESC line are shown that are not altered in $\beta$ catenin knock-out to wild-type ESCs (ID1, ID2, ID3, etc.). The light green circle in the middle reports about all upor down-regulated mRNA transcripts in $\beta$-catenin homozygous knock-out ESCs. Very important stem cell genes like TERT are down regulated to undetectable levels, others are much reduced like Nanog, Klf-2, Dppa4, Dppa-5, and many more (see figure 3). However, and very unexpectedly, there are also some genes whose expression seem to be slightly altered in heterozygous $\beta$ catenin knockout cells despite unaltered protein level and most overlap with those that are downregulated in homozygous $\beta$-catenin knockout cells: e.g. Gbx-2, Nr5a2, SP5, Rex-1 are down-regulated in heterozygous and homozygous $\beta$-catenin knockout cells but stronger in the double mutants, while Plakoglobin is upregulated in both, homozygous and heterozygous ESCs. This can be maybe not explained by a dosage effect, as $\beta$-catenin protein levels are strikingly unaltered and there is no significant detectable change as measured by a classical immunochemical protein western blotting. Furthermore, Nanog and Ephrin B2 are upregulated in these heterozygous ESCs. The changes are very slight but could be real and relevant in theory but this would raise many difficult questions. How could $\beta$-catenin or CTNNB1 control the fine-tuning of these genes if the protein level is unaltered? The answer to this new scientific question carves out a new hypothesis but cannot be fully solved here. One explanation can be slight changes in the posttranscriptional modifications, i.e. PTM isoforms, that are needed for the steady state but which affect the transcriptional machinery too. Better methods can also become new gold standards, but a semi-quantitative qPCR is not always better than a semi-quantitative RT-PCR.

\begin{tabular}{|c|c|c|c|}
\hline Gene & Forward primer $\left(5^{\prime}\right.$ to $\left.3^{\prime}\right)$ & Reverse Primer $\left(5^{\prime}\right.$ to $\left.3^{\prime}\right)$ & ict (bp) \\
\hline Axin2 & GGATACCAAAACTTTGCGAGTGGCC & CGGTCTGTGCCTGGTCAAAC & 160 \\
\hline Dnmtrl & GCTCTAAGACCCTTGAAACCTT & GTCGGTTCACTTTGACTTCGTA & 213 \\
\hline Dppa5 & ATGATGGTGACCCTCGTGAC & ACCTCGATAAGTTCTTCGGGAG & 209 \\
\hline Dppa3 & GACCCAATGAAGGACCCTGAA & GCTTGACACCGGGGTTTAG & 130 \\
\hline EphA2 & GCACAGGGAAAGGAAGTTGTT & CATGTAGATAGGCATGTCGTC & 129 \\
\hline EphB2 & GCGGCTACGACGAGAACAT & GGCTAAGTCAAAATCAGCCTC & 230 \\
\hline Esrrb & GTGTTCCTCATCAACTGGGCC & AGTCCTCGGCGTATGCCAGC & 160 \\
\hline Fgf5 & CGTTTTCTTCGTCTTCTGCC & TTAACACACTGGCTTCGTGG & 198 \\
\hline Galnt16 & AGGTGCTGTGAACAACATTAG & CCCAGAAGGGTTTCAGCTCAG & 137 \\
\hline Gbx2 & GCACTCAACTCAAAAAGCCA & TACGTGCCCAAAGGTAAACA & 108 \\
\hline Gelsolin & ATGGCTCCGTACCGCTCTT & GGCTCAGACACCCGACTTT & 134 \\
\hline Hprtl & GCTGGTGAAAAGGACCTCT & CACAGGACTAGAACACCTGC & 249 \\
\hline Kif2 & CTCAGCGAGCCTATCTTGCC & CACGTTGTTTAGGTCCTCATCC & 123 \\
\hline Nanog & CCTGATTCTTCTACCAGTCCC & GGCCTGAGAGAACACAGTCC & 123 \\
\hline $\mathrm{Nr} 5 \mathrm{a} 2$ & TGAGGAACAACTCCGGGAAAA & CAGACACTTTATCGCCACACA & 242 \\
\hline Oct6 & TCGAGGTGGGTGTCAAAGG & GGCGCATAAACGTCGTCCA & 205 \\
\hline Plakoglobin & TGGCAACAGACATACACCTACG & GGTGGTAGTCTTCTTGAGTGTG & 135 \\
\hline Sox2 & GCGGAGTGGAAACTTTTGTCC & CGGGAAGCGTGTACTTATCCTT & 157 \\
\hline Sp5 & TGGGTTCACCCTCCAGACTTT & CCGGCGAGAACTCGTAAGG & 195 \\
\hline $\mathrm{T}$ & GCTTCAAGGAGCTAACTAACGAG & CCAGCAAGAAAGAGTACATGGC & 117 \\
\hline Terc & CCAGCGGGCCAGGAAAGTCC & GCCCCGCGGCTGACAGAGGCGA & 121 \\
\hline Tert & CCCACCGCACCTCATGGA & CCCCGGGCTGCTGCGGAGC & 244 \\
\hline Vinculin & TGGACGGCAAAGCCATTCC & GCTGGTGGCATATCTCTCTTCAG & 136 \\
\hline Zfp42 & TGGAAGCGAGTTCCCTTCTC & GCCGCCTGCAAGTAATGAG & 128 \\
\hline Zic2 & CTGCAGCCGCTGCGGCCGCG & GCGCCGGGTCCGAAGAGCCC & 160 \\
\hline
\end{tabular}

Figure 4: Primers used for semi-quantitative assessment of mRNA biomarker levels for ESCs or iPSCs.

In 2008-2009, this panel of primers was used to extend our pre-existing panel $[2,15]$ and heterozygous $\beta$ catenin ESCs were compared to wild-type ESCs and double-knockout $\beta$-catenin ESCs but its publication was much delayed due to a project stop. Figure 2 gives an overview of the cell biological phenotype of the different cell lines tested and via IHC for $\beta$-catenin and E-cadherin, showing a pronounced cell adhesion defect in $\beta$-catenin and E-cadherin KO ESCs and tightened colonies in hyperactive $\beta$-catenin ESCs (see Figure 2 and legend for more detail).
It could be possible that a different quantity level of $\beta$ catenin isoforms with slightly different post-translational modifications (PTMs) of $\beta$-catenin is the cause of this effect, termed PTM-isoforms. If there is only one allele expressed, transcripts seem lower, and there maybe PTMs in play that extends the half-life of $\beta$-catenin to enable its accumulation to the same cell-physiological amounts. This would be a re-adjustment to achieve normality and mechanisms would be in constitutive molecular action to achieve this, in a less passive state of kinases, deacetylases, phosphatases, glycosylases, one could test 


\section{Journal of Embryology \& Stem Cell Research}

this hypothesis via MS one day. There are less redundancy and more active molecular work to be done, hence a second mutation, even a heterozygous-recessive one, of a different developmental gene, could have an effect on the signaling level and promote hidden or dormant (lower network stability type) $\beta$-catenin phenotypes that slightly appear in the slight marker changes in Figure 3 of $\beta$ catenin (+/-) ESCs.

Now, there are two effects: (i) predominantly the markers are downregulated like in the homozygous KO ESCs (Figure 3 olive green overlap): Gbx-2, Sp-5, Rex-1, Plakoglobin, or EphA2 - this indicates a lower level of $\beta$ catenin activity in the nucleus, maybe due to the PTMs or concentration; and (2) some genes are contrarily regulated, in the opposite direction of the homozygous KO LOF of $\beta$-catenin (-/-), like EphrinB2 or Nanog. These slight imbalances on the biomarker-level were often overlooked but could give important clues about promoter regulation. For example, Nanog expression seems to depend on $\beta$-catenin in a more complex way than was previously anticipated. The level or PTM isoforms seems to play a role at transcription. Loss of $\beta$ catenin clearly reduces Nanog but a PTM-reestablishment seems to activate it (see Figure 2). This would indicate that PTMs of $\beta$-catenin can be in play in the regulation of Nanog, and also a block in exon 3 PTMs has a very slightly positive effect. Hence, $\beta$-catenin plays a role in transcription and TCF/Lefs could play a minor role, while a mechanism that assures homeostatic $\beta$-catenin levels, a mRNA-corridor or level-precision-system, makes use of PTMs that slightly affect the transcriptional activity of its targets. Usually, this won't have an effect, and signaling, transcription and adhesion function normally, but in situations of additional perturbations, e.g., double knockout $(+/-\beta$-catenin, $+/$ - of a somehow interacting developmental or signaling genes) bottlenecks can appear. As I also suggested before, PTM isoforms play a role in ESC-stemness and differentiation [23].

Before we go into the detail of the individual marker changes in the ESC phenotypes, biomarkers and pluripotency regulators that are either up- or downregulated, it is important to note that the changes are to be interpreted as a raw data matrix that is to be reconstructed into a transcriptional logic program with circuits, very fare comparable to a diffraction pattern that gives rise to a protein structure. This is of course very different, but the idea is that many mechanisms of action and subsequent regulations are in play that are either direct or indirect at different levels, and the markers are an end-point measurement. In the future, dynamic biomarker assessment will further help to draw the circuits and mechanisms. Also, the more ESCs lines are and will be tested in a standardized experimental way, the better the interpretations will get, thus an international iStemCore systems biology hub is proposed (Figure 1). At the same time, more jobs and fair and sustainable career path are still much needed in science in order for anybody to do a good job.

\section{Further Dissecting $\beta$-catenin in Stemness}

The sum of these data in Figure 3 and 5 already starts the bigger biomarker discussion to reveal the bigger picture of $\beta$-catenin in ESCs. These preliminary results cannot finalize the discussion but give an open system biology idea of what could be discussed in iStemCore projects and the iStem international research programs and open access platforms (Figure 1). The changes are briefly commented, interpreted and summarized here. Finally, an updated model is derived and suggested to further refine the overall big picture of $\beta$-catenin in ESCs. Biomarker interpretations aim at a better global understanding of the transcriptional logic behind the stemness state, the ground state, and adjacent states, as well as the biomedical mechanisms of natural in vivo and in vitro stemness and differentiation, and what differences might exist, or to find out how artificial an in vitro differentiation can get and how much in vivo resemblance is needed for differentiation [1]. Globally interpreting the changes summarized in Figure 5, most changes in stemness and biomarker genes have occurred in $\beta$-catenin LOF (-/-) ESCs, the second most changes in stemness and biomarker genes are co-regulated by Ecadherin and $\beta$-catenin (see overlap), and the third highest amount of stemness marker genes are only found in E-cadherin KO (-/-) ESCs or are only found in $\beta$-catenin GOF (delta-exon3, dominant heterozygous; hyperactive isoform) ESCs Figure 5.

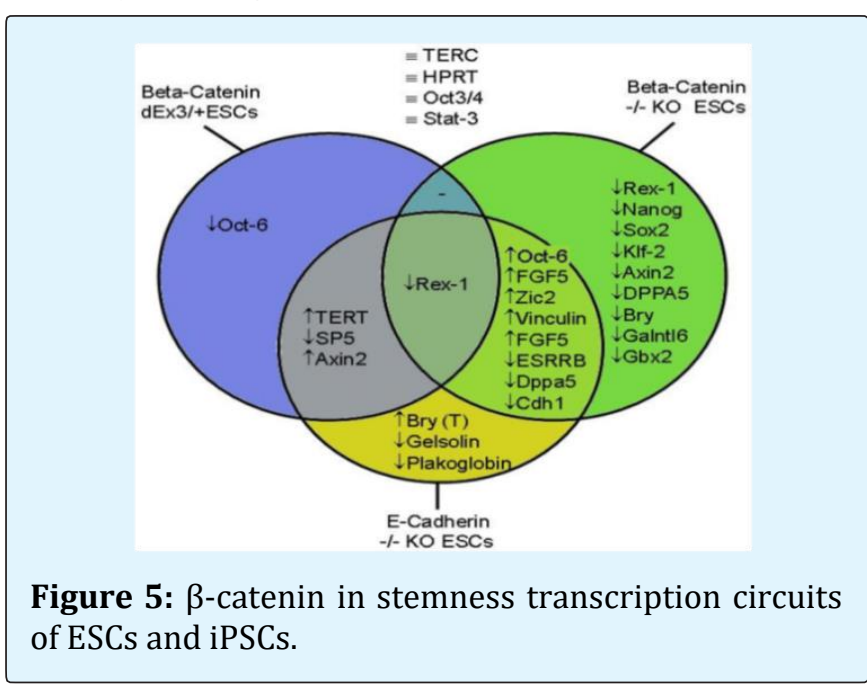




\section{Journal of Embryology \& Stem Cell Research}

Four ESC lines, $\beta$-catenin GOF and LOF $(-/-,+/-)$, and E-cadherin LOF, were compared to each other as a proofof-principle concept for the systems biology approach. The overlap in the expression changes of the stemness biomarker and pluripotency regulator expression patterns is indicated as overlapping circles in Figure 5. Up- and down-regulated biomarker genes are indicated in comparison to wild-type ESCs with upwards or downwards arrows, respectively. Supplementary Figure S2 is a preliminary basis to continue with systems biology, omics, qPCR, and new cutting-edge methods and approaches, with the goal to enable standardized and compatible, i.e. comparable, contributions on one platform, e.g. the iStemCore (Figure 1).

Interpretations are to be viewed in the ESC stemness network [3-7] and every change of biomarker genes can be due to the promoter DNA/MPC composition. The molecular mechanisms that are found in ESCs/iPSCs can also play a role in other cells. The cell adhesion effect in Ecadherin $\mathrm{KO}$ and $\beta$-catenin KO ESCs is accompanied by slight cytoskeletal changes, for example in F-actin (see Supplementary Figure S3). Of note aside, I was hindered to continue my projects whenever I became successful,, in each and every case, project and position, also here, when I found that TERT and telomeres are lost in $\beta$-catenin ESCs (Figure S2 A, C). Additionally, I found that $\beta$-catenin binds Oct-3/4 MPCs and EIF6 mRNA complexes with key target genes like Nanog or Klf2 and Zfp42 (see Supplementary Figure S4). These preliminary findings indicate a new mechanism of regulation by $\beta$-catenin to be studied more systematically. Can the EIF-MPC explain the loss of Nanog protein? To better interpret and summarize all findings, every biomarker change will be explained and discussed in more detail here:

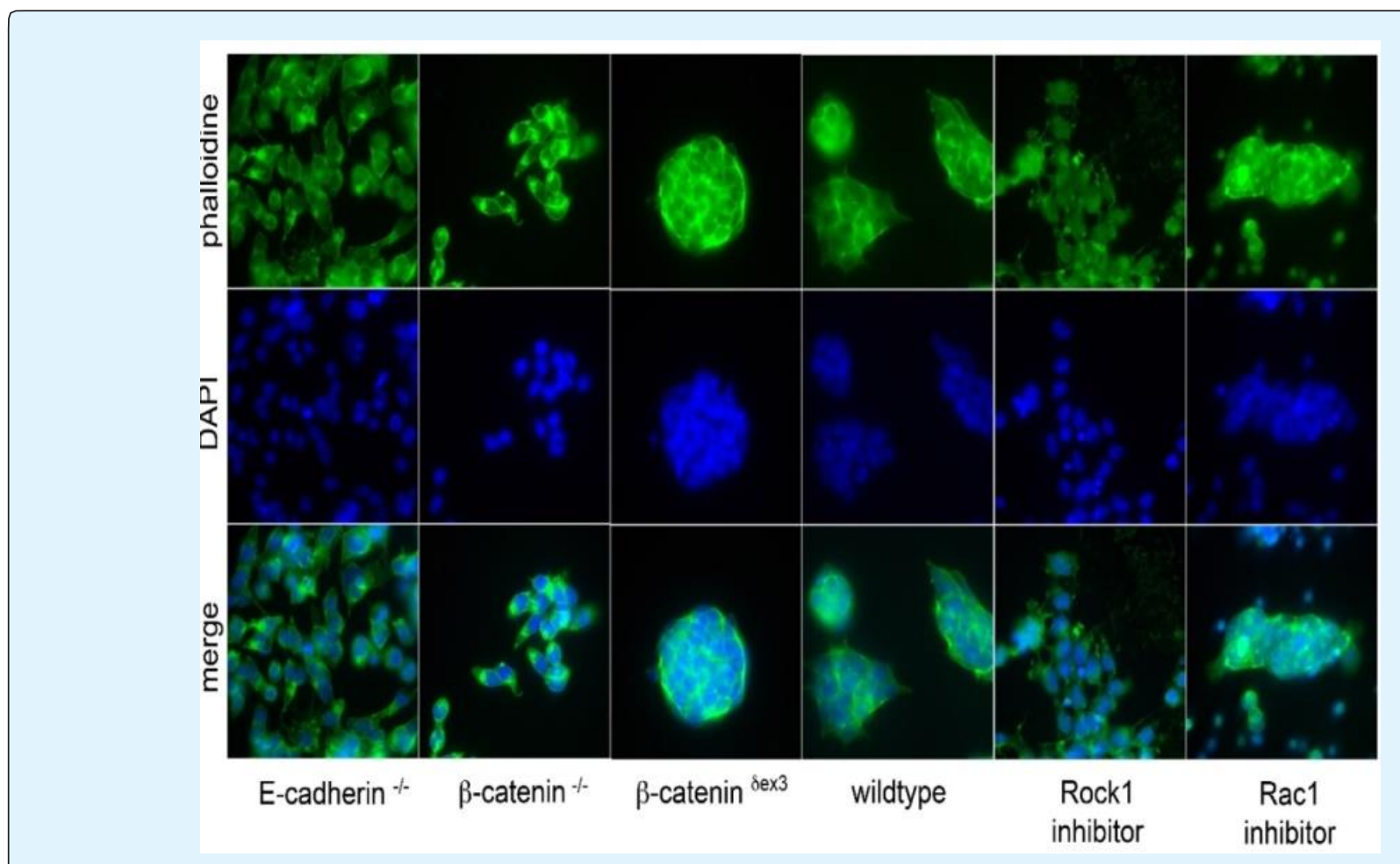

Figure S3: Phalloidine staining in the mutant ESCs reveals slight changes in polymeric F-actin (cytoskeleton), especially in $\beta$-catenin KO (-/-) and E-cadherin KO ESCs (-/-) lacking cell adhesion. 


\section{Journal of Embryology \& Stem Cell Research}
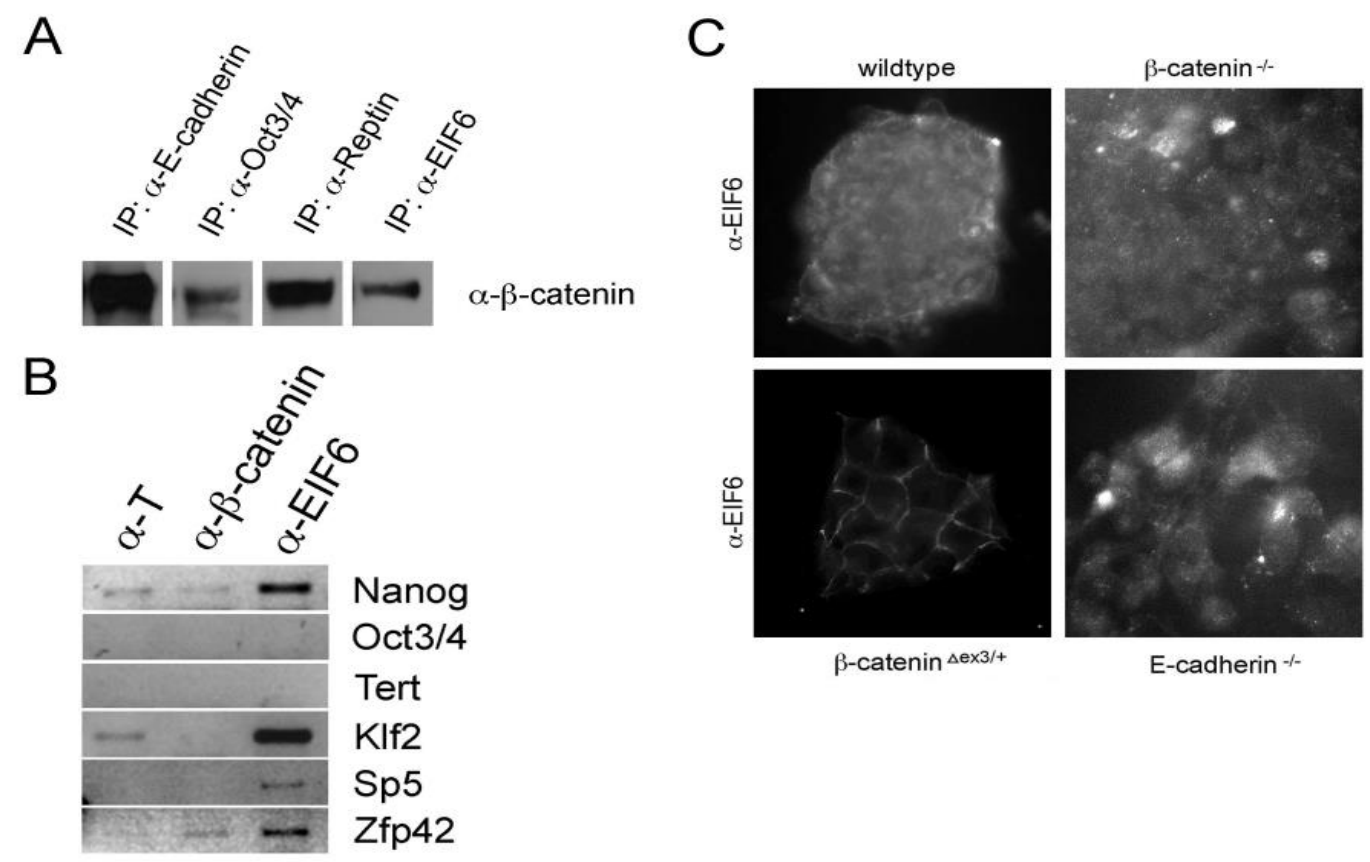

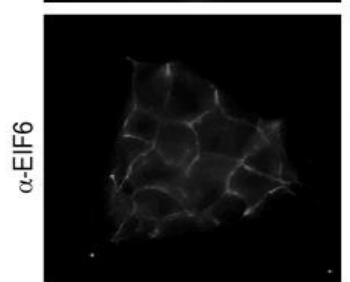

$\beta$-catenin $\Delta \mathrm{ex} 3 /+$

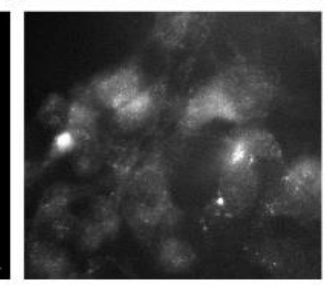

E-cadherin $\%$

Figure S4: $\beta$-catenin could have additional functions with EIFs and in mRNA. (A) RIP with $\beta$-catenin antibody binds EIF complex of factors, including EIF6 (C), which bind to some of the target mRNAs (B).

\section{0ct-6}

A very striking change in the regulatory response pattern can be seen for Oct-6, the official gene name of this octamer transcription factor is Pou3f1 (POU domain, class 3 , transcription factor 1 ), a member of the Oct-family of POU domain transcription factors, just like the more prominent Oct-3/4 factor. Like most stemness and pluripotency genes, the Oct- 6 promoter is occupied by and regulated by 0ct-3/4 [24]. Oct-3/4 has an activator and repressor domain that can be used for switching events of MPCs that are potentially containing $\beta$-catenin [2] and Smads the TGF $\beta$ signaling mediators. An early important work by Ben-Shushan 1998 [24] has revealed that the occupation of Oct-3/4 (and Sox-2) is a molecular platform of activation and repression that could serve thousands of genes in the genome and Oct- 6 might be the game changer and the question is what pulls the trigger between activation and repression. $\beta$-catenin seems to be much involved in Oct- 6 repression, which is up-regulated in $\beta$-catenin LOF (-/-) and in E-cadherin LOF (-/-) ESC lines, while it is down-regulated in $\beta$-catenin GOF ESCs, the hyper-activated form devoid of an exon-3-related destruction box that hyper-activates TCF/Lef-1 Topflash reporters, comparable to GSK3-inhibition or Wnt3a activation in ESCs [5]. Oct-6 is a biomarker member of the epiblast-derived syn-expression group of the epiblast stem cell (epiESCs) state and is up regulated in conjunction with factors like Fgf-5 [5] or Zic-2 [25] that will be mentioned later in this list of biomarker interpretations. With respect to $\beta$-catenin, this Oct-6 marker already drafts the story of four intricate regulatory branches of how $\beta$-catenin regulates a huge spectrum of direct or indirect target genes (see Figure 6).

\section{Four Regulatory Branches of $\beta$-catenin in ESCs}

(1) $\beta$-catenin acts repressive on the Oct- 6 promoter, which is shown by GOF and LOF. (2) The GOF effect could be direct or indirect, which could be TCF or non-TCF dependent. (3) LOF of E-cadherin, the official gene name is $\mathrm{Cdh}-1$, is also known to activate $\beta$-catenin, as it can sequester and buffer its concentration and could have a direct effect, via TCF or non-TCF, or E-cadherin/EMTfactors like snail [20]. E-cadherin anti-EMT signals could block the mesenchymal stage or state via factors of the transcription network [20] that can be partially linked to the differentiation state in ESCs and iPSCs. (4) $\beta$-cateninPTM-isoforms could have a more special effect compared to pure accumulation of $\beta$-catenin. Different PTMisoforms arise during signaling, EMT and loss of Ecadherin, or presumablyy also in heterozygous $\beta$-catenin 


\section{Journal of Embryology \& Stem Cell Research}

KO (+/-), which still has to be further shown, characterized and verified. $\beta$-catenin's interaction with $\mathrm{E}$ cadherin on the cell membrane also buffers and binds a significant amount of free and active $\beta$-catenin. This is one of the reasons why it acts as a tumor suppressor gene, also by stabilizing the epithelial phenotype and thereby reducing potential metastasizing cell biological effects.
These are the four main branches and general modes of action of $\beta$-catenin in ESCs, iPSCs and all other cells and tissues with respect to the endogenous promoter effects. These activation modes could be revealed for Oct- 6 and were subsequently modeled for all $\beta$-catenin target genes in Figure 6 to reveal the pattern.

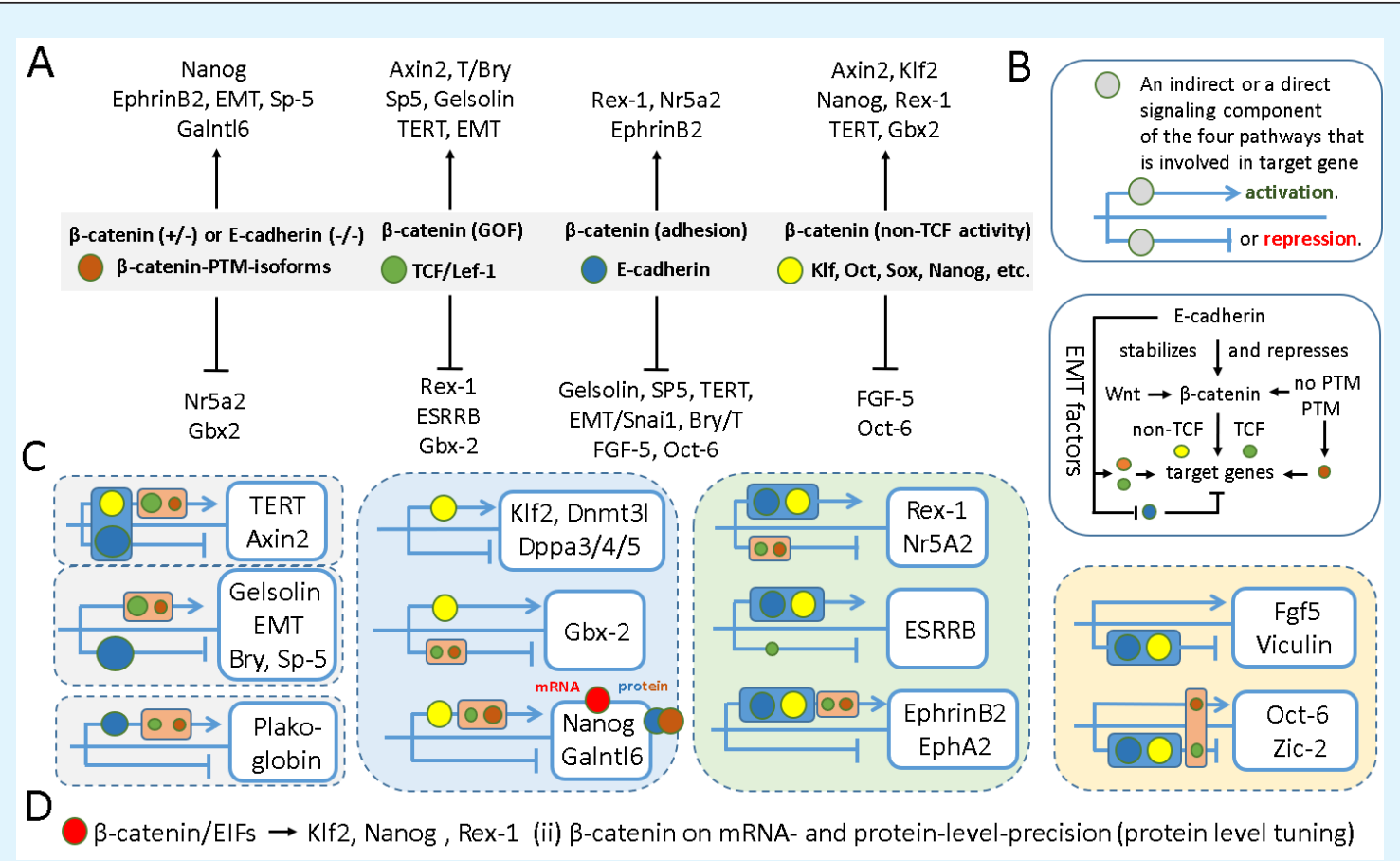

Figure 6: Schematic diagram drafting four main routes of target gene regulation by $\beta$-catenin. Another way of regulation could happen directly on the mRNA-level, for example via EIFs. Visualization and graphical representations are a key element of systems biology and may include many different models and drafts of different levels of abstraction and evidence. Attention, this figure shows the endogenous promoter effect diagram, which is one layer above the direct molecular factor binding promoter map of systems biology. A general promoter map is drafted for all target genes to reveal general mechanisms and patterns: four groups were found of potentially seven. (A) Abstracted simplification into four pathways branches of $\beta$-catenin's mode of action on target promoter activation (arrow) and repression (stop arrow): specific $\beta$-catenin PTM-isoforms (brown circle), TCF/Lef-1 (green), E-cadherin (blue) and non-TCFs (here stemness factors: Klf, Oct, Nanog). (B) Legend and simplified overview of the general effects of $\beta$ catenin via TCF, non-TCF, and EMT, non-TCF and EMT related target gene regulation. (C) Direct and indirect effects on natural promoters (TCF- $\beta$-catenin or KLF4- $\beta$-catenin is a direct interaction, while E-cadherin's EMT-factors could be indirect or direct via a proteolytic Cdh1-tail and $\beta$-catenin). The size of the circles indicates a bit the strength of the direct or indirect effect or affect. The patterns on the promoters can reveal a new mechanism and motive of transcriptional and epigenetic regulation, and allows a classification into responsiveness groups of four consolidated mechanisms. Promoter effect pattern (blue): a striking combination of non-TCF- $\beta$-catenin and an E-cadherin-factor on key promoters. Promoter effect pattern (palm-color): a combination of a $\beta$-catenin PTM-isoform and TCF/-Lef1activation. Please note that the Nanog protein level is reduced in $\beta$-catenin KO cells and amending RT-PCR results are found in the Supplementary Figure S2. (D) There might be additional layers of regulation how $\beta$-catenin might affect target genes: there might be seven general principles of regulation. One of them could be on the RNA/protein level by interacting with the EIF complex. Klf2, Nanog, and Rex-1 mRNA or protein could be regulated in EIF RNA MPCs (see Figure S4) in summary; this reveals the 4+1 major routes of regulation, and scarcely the literature has also revealed potential additional ways that need to be researched in more detail in a systems biology approach.. 


\section{Journal of Embryology \& Stem Cell Research}

\section{FGF-5}

Like Oct- 6 and Rex-1, FGF-5 is part of the epiESC biomarker syn-expression group. Once the ESCs transdifferentiate from the ICM-like stem cell ground state into the next adjacent transcriptional states of in vitro epiESCs, the pluripotency and stemness marker Rex- 1 is reduced and lost, while FGF-5 increases, in conjunction with Oct- 6 [5]. Thus, a syn-expression mechanism seems to exist. Interstingly, part of this molecular synexpression state is also reached in E-cadherin KO ESCs, an important new finding. This and synexpression also reveals the importance of several layers of promoter mapping like the promoter effect circuits in Figure 6 for systems biology and the iStemCore project.

\section{Rex-1}

Rex-1 is a widely used key stemness biomarker that has been shown to be regulated by $\mathrm{Wnt} / \beta$-catenin signaling (Anton et al., 2007) [5]. It is reduced in ESCs with a LOF and even in the heterozygous $\beta$-catenin mutants (see Figure 3 and 5) due to up regulation of Oct-6, a Oct-6, a switching event for Oct-3/4 [24], and in the GOF setting it can show a slightly lower level. This would again indicate that (i) $\beta$-catenin is likely directly involved and that (ii) PTMs of $\beta$-catenin are potentially inhibiting the Rex-1 promoter, and (iii) that E-cadherin also] stabilizes the integrity of this specific branch of the crucial stemness network like $\beta$-catenin. Both, E-cadherin and $\beta$-catenin gatekeep the epiblast stage in vitro, i.e. the epiESC state, hence both are pluripotency regulators.

Zic-2: The role of Zic-2 is not fully explored in ESCs and iPSCs. This Zic family transcription factor is also implicated in self-renewal of tissue stem cells, e.g., adult stem cellsin postnatal olfactory bulb neurogenesis, i.a., and tumor-initiating cells in hepatocellular tumors. Interestingly, the major acting transcription factors shift in mouse ESCs from Sox-2 and Oct-3/4 to Zic2 and Otx2 in epiblast stem cells [25]. This epiESCs pattern is also recapitulated in $\beta$-catenin LOF (KO, -/-) cells and is another part of the syn-expression group of Rex-1, Oct- 6 [23] and FGF-5 [2].

\section{TERT}

TERT assures the chromosomal stability in ESCs and iPSCs by extending telomeres via the RNA factor TERC, which is normally expressed in the GOF and LOF ESC lines (Supplementary Figure S5), i.e. the rate-limiting factor is TERT and not TERC in $\beta$-catenin KO ESC telomere maintenance. The TERT promoter is regulated in the $\beta$ catenin GOF and LOF setting and represents an example of TCF-independent transcription in ESCs and thus (a scientifically legitimate assumption) also in iPSCs $[5,15]$ (see Figure 3 and 5). This chromosomal telomere elongation is $\beta$-catenin dependent $[5,15]$ and positively effects Terra lncRNA [26], which is also activated by the Wnt/ $\beta$-catenin signaling pathway and has a stemness and self-renewal effect in ESCs (and thus presumably also in iPSCs) and in $\beta$-catenin GOF scenarios [26].

\section{RT-PCR}

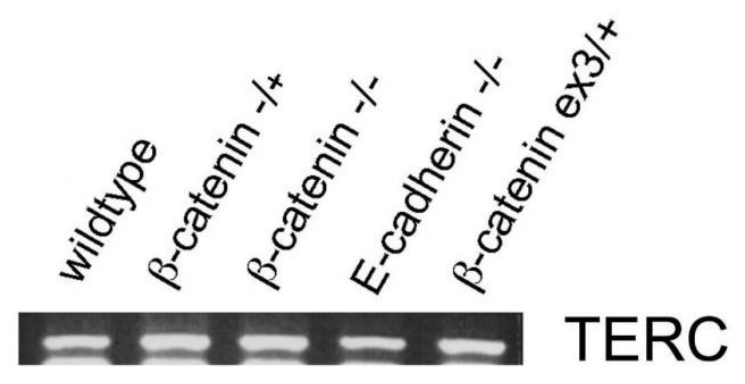

Figure S5: TERC, a required RNA component of TERT is expressed in all cell lines tested, including $\beta$-catenin GOF, LOF, and E-cadherin LOF ESCs. TERT, not TERC, is the rate-limiting factor in $\beta$-catenin KO ESC telomere maintenance that is lost, which remains correct [15].

\section{TERC}

The telomerase RNA component TERC is expressed in ESCs (see Supplementary Figure S5). It is not a limiting factor for Telomerase function in $\beta$-catenin KO ESCs and is only slightly regulated. Maybe there is a slightly higher level of TERC in $\beta$-catenin KO (-/-) and E-cadherin KO (-/-) ESCs due to an active $\beta$-catenin-PTM but this seems to be minimal and thus not relevant for its functioning.

Wnts: Like SP5, Wnt5a and Wnt11 are target genes of canonical Wnt signaling and slightly repress the canonical Wnt/ $\beta$-catenin pathway via its non-canonical branches [5]. Additionally, some Wnts, Frizzleds are changed in $\beta$ catenin KO ESCs [5], which reveals the pattern of regulation that appears in the synexpression group.

Sp-5: The Wnt and $\beta$-catenin target SP5 is reduced in $\beta$ catenin KO ESCs [5] and negatively regulates the Wnt transcriptional program in human pluripotent stem cells [27]. Sp-5 activation by Wnt/ $\beta$-catenin is typical for stem cells with developmental potential and exerts a repressive or modulatory-regulatory differentiating role on $\beta$ catenin. In human ESCs, induction of Sp-5 as a Wnt/ $\beta$ - 


\section{Journal of Embryology \& Stem Cell Research}

catenin target gene stops the $\mathrm{Wnt} / \beta$-catenin transcriptional activation programs of its many additional target genes [27]. $\beta$-catenin seems to activate SP5 which represses its canonical targets in ESCs and thus also in iPSCs. The transcriptional mode of action seems to be repression of normal TCF programs via factors like Sp5, Oct-3/4 and Nanog, and $\beta$-catenin based activation of non-TCF transcription via KLFs, Oct-3/4, Nanog and additional factors $[5,15]$ (see Figure 6). SP-5 is further upregulated by $\beta$-catenin and E-cadherin GOF, which suggests a role to focus $\beta$-catenin on mesoderm target gene induction - but not allone but in a concert with EMT factor.

\section{Axin-2}

Axin-2 is known to be a direct target gene of Wnt/ $\beta$ catenin/TCF2-4/Lef- 1 but could be regulated by non-TCFdependent transcriptional regulation and factors in ESCs [5]. Figure S2 indicates that there is no or only a minimal change in heterozygous $\beta$-catenin ESCs and that the mechanism that establishes the same levels of $\beta$-catenin protein, likely PTMs, seems not to have a strong but maybe a weak effect on Axin2. This is in line with a very classical role of TCF and $\beta$-catenin in transcriptional regulation of its target genes. Nevertheless, Axin-2 is slightly reduced in two separately generated $\beta$-catenin KO cells [5] and Figure 3 and 5. Although a highly sensitive $\mathrm{TCF} / \beta$-catenin reporter (TOP flash, with $8 \mathrm{x}$ or $12 \mathrm{x}$ TCF binding sites) is negative and shows no activity, the Axin2 promoter shows a slight basal activity in wildtype ESCs [5] and Figure 3 and 5 . This can be explained by additional factors that either block a low TCF/ $\beta$-catenin activity on TOP reporters and promoters that simultaneously activate transcription in a non-classical TCF way, e.g. via factors like Oct3/4 [5] and Klf-4 [15] and/or additional factors, like transcriptional regulators. Oct3/4, Nanog, and Klf- $4[5,15]$, Sox and Sal family members, and others could be in play at all such promoters in the context and setting of ESCs and iPSCs, and also in development and disease (see overview in Figure 6). TCF/ $\beta$-catenin can differentially activate transcription if complexed by different interaction factors in MPCs. GOF of $\beta$-catenin, by a delta-exon-3 isoform that lacks the destruction box, which is constitutively phosphorylated by GSK3/CK1 and which also needs Axin1/2 for this ubiquitin-mediated protein degradation [13, 14], clearly activates axin-2 likely in a TCF-dependent manner, as Topflash TCFreporters can also be activated in ESCs (and thus also iPSCs) but more difficult due to the endogenous $\beta$-catenin target gene repressors (Oct-3/4, Nanog, SP-5, Klf-4, and more) [5] and Figure 5 and 6. E-cadherin LOF ESCs yields a higher level of free $\beta$-catenin and a much lower level of overall $\beta$-catenin. Hence, PTM-isoforms and free $\beta$-catenin seem to activate and E-cadherin seems to sequester $\beta$ catenin (isoforms and level) and thereby represses Axin-2 transcription non-TCF factors are also in play (see Figure 6). Up regulation of Axin2 is much higher in Cdh-1 (LOF) and $\beta$-catenin delta-Exon3 (GOF) indicating a dosagedependent effect of $\beta$-catenin also involving TCFs on transcription, i.e. MPCs of several co-regulating factors.

\section{Nanog}

Interestingly, the pluripotency and stemness master regulator Nanog is clearly reduced in $\beta$-catenin LOF (-/-, KO) ESCs, while it is upregulated in heterozygous $\beta$ catenin ESCs (see Figure 3 and 5), likely due to PTM stabilized isoforms of $\beta$-catenin that arise by a proteinlevel stabilizing mechanism that assures same amouts of steady-state $\beta$-catenin likely due to less proteasomal degradation (see Figure 6). It is also upregulated in Ecadherin LOF ESCs due to the higher levels of unbound $\beta$ catenin and also in $\beta$-catenin (dEx3/+) GOF ESCs (see Figure 5). This indicates monoallelic Nanog promoter regulation via TCF- and mainly non-TCF dependent transcription. While Nanog transcripts are still found, Nanog protein level seems to be lost in $\beta$-catenin -/- ESCs, which could be revealed by one in-house antibody in western blotting. This effect could be explained by a mechanism of post-transcriptional regulation, involving EIFs like EIF6 that bind to $\beta$-catenin and Nanog mRNA (Figure S4 and 6D). Figure 6 summarizes the effects on the Nanog promoter, on transcription and translation.

\section{Brachyury (T)}

In this direct comparison, Brachyury, a T-box transcription factor, master regulator, mesoderm marker and specifier of the primitive streak including mesendoderm, is known to be also a $\beta$-catenin and TCF/Lef- 1 co-responsive target that is up regulated by $\beta$ catenin in the context of very early differentiation [2] but not so clearly or only very slightly in ESCs activated by Wnt/ $\beta$-catenin or GSK3 inhibitors, as Bry, aka $\mathrm{T}$, is repressed by the stemness factors (the Oct3/4, Nanog, Sox-2, Klf-4 network) and E-Cadherin, an endogenous factor required for ESC and iPSC stemness, and its subsequent inhibition of EMT [20] that would initiates germ layer formation during the spatio-temporal and regulative primitive streak mesonderm induction. As a result, Bry/T is very strongly upregulated in E-cadherin ESCs (-/-) whiles the very strong GOF of $\beta$-catenin in ESCs, unexpectedly, only results in a comparably small induction of Bry/T-expression. The stemness gene Ecadherin seem to be a major gatekeeper of mesodermal 


\section{Journal of Embryology \& Stem Cell Research}

differentiation, while $\beta$-catenin is a second contextdependent trigger, as I claimed in the earlier study [5]. There is no change in ESCs heterozygous for $\beta$-catenin (see Figure S2 and 6) which indicates that such additional PTMs are not in play. E-cadherin is also a gatekeeper of EMT-factors [20] that can and could drive Bry/T together with context, differentiation stage, and time-dependent differentiation stage level [5]. This context-dependent regulation is explainable with $\beta$-catenin's transcriptional modes and motives of action that were dissected, identified, systematized, and summarized for Bry/T in Figure 6. It seems that several b-catenin target genes have gatekeepers that prevent their transcription in the wrong context: here E-cadherin should be absent for Bry induction by $\beta$-catenin. Sp-5 is upregulated with Ecadherin, maybe to focus the $\beta$-catenin transcriptional program on mesoderm, while still blocking some other targets.

\section{Gelsolin}

Gelsolin, an actin-binding and modulating cytoskeletal protein, is activated mainly in E-cadherin KO (-/-) ESCs and could be repressed by E-cadherin signals (Figure 5, 6). It might be activated by a $\beta$-catenin PTM isoform that preferably bind to and is sequestered by E-cadherin and would be freed in the KO ESCs. However, if $\beta$-catenin isoforms were at play, one would assume an increased Gelsolin expression in $\beta$-catenin GOF or heterozygous ESCs. As this is not the case, it seems to be possible that Gelsolin is regulated in an E-cadherin-dependent manner. Gelsolin is repressed by E-cadherin and E-cadherin is repressed by Gelsolin, and HGF increases Gelsolin that is essential for E-cadherin repression via Snail, Twist or ZEB2, likely via Pi3K and Akt [28]. Thus, Gelsolin could be involved in the molecular mechanisms of EMT $[20,28]$ and E-cadherin in ESCs which drive early differentiation markers like Bry/T.

\section{Vinculin}

Vinculin, is like Gelsolin an actin-binding protein but has a different promoter activity and expression pattern (Figure s2, 3, 5, 6). It matches the pattern of the FGF$5 /$ ct6/Zic2-synexpression group and is inversely correlated with Rex-1. Vinculin is upregulated in $\beta$ catenin $\mathrm{KO}$ and $\mathrm{E}$-cadherin $\mathrm{KO}$ and very slightly also in $\beta$ catenin GOF $(\mathrm{dEx} 3 /+)$. Its downregulation by E-cadherin or TCF-free $\beta$-catenin could be involved in stem cell maintenance, as has been suggested for the effects on ESCs by graphene oxide nanosheets [29], which promote stemness via the downregulation of vinculin. This is especially interesting in that a cytoskeletal protein drives differentiation in ESCs/iPSCs and its gatekeepers are $\beta$ catenin and E-cadherin, which are both and together linking the cell adhesion machinery to the cytoskeleton architecture. This could help to unveil the mechanism of E-cadherin in stemness.

\section{Plakoglobin}

Plakoglobin, also known as $\gamma$-catenin, is known to compensate for the loss of $\beta$-catenin in cell adhesion, and its elevated expression has been associated with this effect ([5] and references herein). It is only very slightly upregulated in $\beta$-catenin GOF and $\beta$-catenin LOF $(-/-$ and $/+$ ) ESCs (Figure S2, 3, 5, 6). This again indicates that the presence of E-cadherin adhesion complex has an impact on the ESC stemness state and thus also the iPSCs stemness state, and on $\gamma$-catenin expression among several additional marker genes. This slight regulation might result in the only very weak compensation of cell adhesion in $\beta$-catenin KO ESCs.

\section{ESRRB}

ESRRB, estrogen-related receptor beta, is part of the reprogramming factors that are activated by Glis-1 [30], like Wnt, EMT [20], Lin28, Myc, and Nanog [30]. The loss of ESRRB in $\beta$-catenin $\mathrm{KO}(-/-)$ ESCs and E-cadherin KO (/-) LOF indicates that there is an important role of $\beta$ catenin and E-cadherin in the reprogramming into iPSCs and in stemness (see Figure 3, 5, 6). In other words, intrinsic $\beta$-catenin is needed as a co-factor and E-cadherin is needed as intercellular adhesion mediator for ESC stemness and to establish pluripotent iPSCs. ESRRB is also known to be regulated be TERRA like Klf-2, which are both thought to be repressed by TCF-3 [27] and activated by $\beta$-catenin in a non-TCF way like Klf- 4 [Figure 5, 6 and $[5,15])$. As a result, E-cadherin and $\beta$-catenin are both intrinsic pluripotency and stemness factors of ESCs and iPSCs as they are required to maintain the transcriptional integrity and functionality. Figure 6 summarizes these effects in the 4+1 modes of regulation. Interestingly, ESRRB is a master regulator of ESCs and a pluripotency factor that can be used to reprogram cells into iPSCs, and upstream of ESRRB, I could identify the E-cadherin/ $\beta$ catenin cell adhesion machinery as a key driver of a complete stemness (see Figure 6). Also, ESRRB and Klf2 are repressed by TCF3 [26] but the upstream role of Ecadherin is unique for ESRRB (Figure S2, 6). Could the described TCF-3 isoform only be functionally repressive [26] in ESCs and iPSCs in the absence of $\beta$-catenin and Terra? TCF-3 was actually found to be very unchanged in $\beta$-catenin ESCs [5]. 


\section{Journal of Embryology \& Stem Cell Research}

\section{Dppa3/4/5}

Dppa4 and 5, developmental pluripotency-associated 4 and 5 are reduced in $\beta$-catenin KO ESCs and Dppa- 5 is also reduced in E-cadherin KO (-/-) ESCs (Figure 3, 5, 6 and [5]). The mechanism of this promoter activity seems to be non-TCF factors that act vial $\beta$-catenin, like Klf-4, Oct-3/4, Nanog, Sox-2, and others.

\section{Gbx-2}

Gbx-2 is co-regulated with Rex-1 and down-regulated in the adjacent epiESC state characterized by the FGF-5, Oct- 6 and Zic- 2 syn-expression group $[5,24]$ (see Figure 5 and 6). It is reduced in homozygous $\beta$-catenin $\mathrm{KO}(-/-)$ and also slightly in the heterozygous knock-out of $\beta$ catenin (+/-). Additionally, Gbx-2 is not controlled by Ecadherin, but seemingly by a non-TCF-dependent $\beta$ catenin route with a slight role of PTM-isoforms found in the heterozygous $\beta$-catenin KO state (see Figure 3, 5, 6).

\section{Sox-2}

Sox-2 mRNA and protein levels are unchanged or only very minimally changed in ESCs with $\beta$-catenin GOF, LOF or E-cadherin KO mutations. Sox-2 and Oct-3/4 are coregulated in a feed-forward loop. Also on the protein level, there are no measurable changes in Sox- 2 in $\beta$-catenin KO, GOF and LOF ESCs.

\section{0ct3/4}

Like for Sox-2, there is no change for Oct-3/4 in all cell lines tested and also on the protein level there is no change. The core stemness Oct-3/4/Sox2 circuit is selfreinforcing in Lif/FBS self-renewal media and Oct-3/4 and Sox form a core regulatory tandem within the stemness circuit (see Sox-2) [5, 24] and they are among the iPSC and core stemness factors [6]. This core circuit is not effected but there are many very important downstream genes under the co-regulation of $\beta$-catenin and E-cadherin, which can be somtimes also upstream (see ESRRB or Rex-1).

\section{Klf-2}

Like ESSRB, Klf-2 is repressed by TCF-3 in ESCs [26] and up regulated by the Wnt target TERRA, which represses this TCF-3 activity [26]. In ESCs that are devoid of $\beta$-catenin (-/-, KO), KLF-2 is much reduced despite a lack of TCF/ $\beta$-catenin reporter activity [2], but the expression is maintained in E-cadherin and $\beta$-catenin GOF (delta-exon-3/+). Maybe the total loss of $\beta$-catenin drives TCFs into hyper-repression? Alternatively, a direct or indirect non-TCF dependent co-activation role of $\beta$ - catenin can be suggested. As discussed for ESRRB, it seems to be a non-TCF cofactors in play and a role of Ecadherin via $\beta$-catenin. This interesting regulatory pattern could also be revealed in Figure 6.

\section{Galntl6}

Polypeptide $\mathrm{N}$-acetylgalactosaminyl transferase like 6 is an UDP-GalNAc glycosyltransferase that transfers sugars from the sugar donor UDP-GalNAc to serine and threonine residues. It is lost in $\beta$-catenin LOF ESCs (Figure 3 and Figure 5) and upregulated in ESCs with a GOF of $\beta$ catenin, slightly weaker in ESCs with E-cadherin KO mutation (-/-) and slightly increased in $\beta$-catenin heterozygous ESCs (+/-). These sugars might play an essential role in the early regulation of stem cells.

\section{Nr5a2}

The nuclear receptor subfamily 5 groups A member 2, $\mathrm{Nr} 5 \mathrm{a} 2$, is a pluripotency factor, controls neural cell fate, , acts as a central node in the regulatory networks, and in ESCs it separates a mitochondrial from a cytosolic function of ribosomal gene expression [31]. It drives pluripotency and stemness in ESCs and iPSCs and is repressed by EMT [20] and $\beta$-catenin (see Figure 3, 5, and $6)$. Its regulation by $\beta$-catenin could be comparable to Rex-1 in that it is activated by E-cadherin and via a nonTCF-dependent role of $\beta$-catenin. It adds to a list of important stemness factors that are controlled by Ecadherin and $\beta$-catenin and thereby shows that Ecadherin and $\beta$-catenin are key stemness factors. This brand new pattern of target gene regulation via $\mathrm{E}$ cadherin and non-TCF $\beta$-catenin co-factors seems to be striking in ESCs and iPSCs, and could be researched in more detail of the promoter and the type of pathway (like Rex-1, Klf-2, etc., see Figure 6). Aside, the partial loss of some $\mathrm{Nr} 5 \mathrm{a} 2$ expression level in heterozygous $\beta$-catenin cells could additionally indicate a role of some PTM isoforms on its promoter that are altered in these ESCs.

\section{Dnmt3l (Dnmtrl)}

DNA (cytosine-5- ) methyltransferase 3-like is a transcript that has been often found in Lif-cultivated mouse embryonic stem cells in expression screenings (NCBI blast data). Sirt-1, nicotinamide adenine dinucleotide (NAD)+-dependent deacetylase, a histone deacetylase, is like TERT a lifespan extending enzyme and regulates DNA methylation and differentiation potential of ESCs by antagonizing Dnmt3l [32]. Sirt-1 deacetylates and activates $\beta$-catenin in the nucleus and Sirt-1 KO ESCs $(-/-)$ highly express Dnmt3l, while Sirt-1 interacts and inhibits Dnmt3l protein [32]. Dnmt3l (Dnmtrl) likely 


\section{Journal of Embryology \& Stem Cell Research}

represses germ line developmental imprinted and germ line genes via methylation and mediates much of the knockout effect of Sirt-1 via its de-repression. Dnmt3l could mediate the epigenetic and transcriptional repression of imprinted and germ line developmental genes in Sirt-1 depleted ESCs [32]. Sirt-1 also activates Nanog and controls p53 [32], while Lif-Stat3 activates Dnmt3l that hypermethylates and represses differentiation genes likely via Dnmt3a a reader and effector of the epigenetic stemness state. Hence, Dnmt3l mediates Lif/Jak/Stat in the maintenance of the stemness network [33]. Generally spoken, it is regulated by stemness factors and by $\beta$-catenin mechanisms that resemble the one of Dppas and Klf2. Loss of Dnmt3l in $\beta$ catenin KO ESCs (see Figure S2, 3, 5, 6) can be a direct cause why some peripheral stemness markers are changed in the various GOF and LOF ESCs analysed in this study.

\section{HPRT}

A control marker, hypoxanthine guanine phosphoribosyl transferase; like GAPDH or actin. Several normalization controls can normally also show very slight differences that might be minimal and not relevant. When controls are totally equal they are often fake in the plethora of scientific literature, which is a serious topic also for systems biology questions and science in general. Systems biology should find a global way, or several alternative good ways, to normalize the omics, individual, and validation data for iStemCore database compatibility (Figure 1) and for a better comparability of all data sets and the characterization of iPSCs and ESCs, including the gene editing rescues and analytical or therapeutic assays.

\section{Discussion}

This kept-simple proof-of-principle preliminary systems biology study of 2008-2009 already helped to pave the way for the iStemCore (Figure 1) and fair and open international systems biology platforms in general $[8,19]$. It was also successful in further revealing many additional important molecular coherencies and molecular mechanisms of key biomarker dynamics of the stemness circuits of iPSCs and ESCs - and could indicate the need of open science $[8,19]$ and standards for method, materials, information and reading and writing access for data, models, and publishing (including postdoc rights). Many interesting studies have revealed and elucidated the stemness factor network and circuits $[3,4,5,9,15]$, this study, and many more interesting and outstanding publications that can be systematically listed in the iStemCore IT-platform in a findable and orderly way. A fair free and open collaborative open science $[8,19]$ platform, however, is still elusive today, and it is still not clear if and when an international hub, like the iStemCore will be created (Figure 1).

Characterizing GOF and LOF KO ESCs is an important and powerful first-line systems biology strategy to reveal and better dissect the role of all genes and gene products in the long-phase post-genomics era. It can help to understand the underlying molecular mechanisms that govern the stemness state and the differentiation into all celltypes and tissues of the body - "drawing the roadmaps for bioengineering".

This work suggests a specific hub for ESCs and iPSCs and the iStemCore to steadily advance regenerative medicine and stem cell research in a continuous and systematic way to help research to build on each other in an orderly way that properly stores and delivers all information and materials, including bio- and databanking, IT, ICT and programming services for the laboratory scientists as an always division of labor, and models, crowd-sourcing, an access and contribution hub and open platform for publications, interactive discussions, with open access reading and writing rights for all postdocs no matter if faculty or not. Sustainable research career paths are needed like systems biology service positions. Laboratory or theoretical researchers should not need any programming skills as the programming and bioinformatics tools would be free to all scientific users that work on the scientific finding together in a more standardized or systematic way. A knowledge database helps in the verification and falsification, both are good and needed, like positive and negative findings, and the most important thing is that we move forward, together and steadily.

With regard to the role of $\beta$-catenin in ESCs, this work suggests that the multi-functionality of genes is another reason that makes systems biology approaches so important and $\beta$-catenin has extended the one-gene-onefunction hypothesismany times and early on. $\beta$-catenin not only has a dual role but can even interact with several interaction factors to regulate and mediate several functions in cell adhesion and different ways in transcriptional co-activation. Thereby this pilot work also illustrates the many challenges to open science systems biology platforms like the iStemCore to incorporate all qualitative and quantitative findings (that are today often still semi) in a systematic platform approach. Ways to graphically summarize findings will be needed like 


\section{Journal of Embryology \& Stem Cell Research}

mapping promoter binding data, promoter effect data, and circuits, inclusive for all researchers on the topic.

Figure 6 summarizes the findings on the endogenous promoter and mRNA transcript level that is caused by experimental perturbation of gene function via GOF (gain of function; delta-exon-3 $\beta$-catenin, a hyperactive form that escapes PTM-driven proteasomal degradation), homozygous LOF ( $\beta$-catenin knock-out ESCs; -/-), heterozygous ( $\beta$-catenin knock-out ESCs; $+/-)$, and LOF $\mathrm{KO}$ of E-cadherin, the major protein interaction factor that mediates cell-to-cell adhesion via a $\beta$-catenin cytoskeletal linkage. All target genes of $\beta$-catenin could be subgrouped and classified as one of the four (i-iv) mechanisms, while additional interaction factors might be in play, like EIF-mRNA-MPCs (Fig. 6 D and Figure S4). This still very preliminary EIF6 or EIF-RNA-MPC effect on Nanog (see Figure 6, S2) could be further studied and can be maybe different, however, additional mechanisms of regulation seem to be involved on the control of a specific mRNA and protein level.

Figure 6 schematically represents the finding of Figure 3 and 5 by summarizing and interpreting Figure S2. For all four mechanisms (i-iv), the observation can be made that a bidirectional, positive activating and a negative repressing, direct or indirect, influence on target genes can be found, illustrated by an arrow or T-end arrow in Figure 6. This can be unexpected, as transcriptional activators and repressor are usually thought to have separate functions, but also 0ct-3/4 has repressor and activator domains [24], and theoretically activation and repression can be mediated by binding, cofactors, MPCs and epigenetic factors. All four mechanisms (i-iv) act bidirectional and the molecular mechanisms that can switch an activator into a repressor would be interesting to find (e.g. epigenetic modifiers or co-activators and repressors), as this seems to be the mechanism that assures the proper mRNA level, not to much not to little, a balanced corridor, also for proteins and even $\beta$-catenin itself, as shown in the heterozygous KO ESCs, which is also an example of this regulation that could be to some extent a self-regulation.

Non-TCF mechanisms of $\beta$-catenin on genes like Rex- 1 , TERT, Dppa4/5, Klf-2, among others, are and were one of the most important findings [5, 15 and Figure 5 and 6]. The role of factors like Oct3/4 and Klf-4 $[5,15]$ and their target genes can be further studied. However, a second important finding was not reported: the role of $E$ cadherin in stemness and pluripotency, and the role of PTM-isoforms and regulation via TCFs and non-TCFs that can be in conjunction with E-cadherin. TCF-reporters are less responsive in ESCs as compared to HEK cells [5], as TCFs are repressed by factors like Oct-3/4 and Nanog and $\beta$-catenin is shifted in mouse ESCs/iPSCs towards TCFindependent transcriptional activation, e.g. via KLF-4 and factors like Oct-3/4, i.a. But $\beta$-catenin target genes like TERT (Figure S2, 5) [15] are not only regulated by nonTCF, i.e. KLF-4 [15], as E-cadherin KO ESCs show elevated TERT transcripts (Figure S2, 6) and a much lower $\beta$ catenin protein level. Also, hyperactivated $\beta$-catenin also drives TERT mRNA level. This indicates that free and active $\beta$-catenin can further drive the TERT promoter, PTM-isoforms have a very slight effect and E-cadherin seems to gatekeep excess of TERT mRNA like an ideal tumor suppressor, which blocks: (a) EMT, (b) hyperTERT- expression, and (c) oncogenic $\beta$-catenin overall protein level. $\beta$-catenin interacts with KLF4 on the TERT promoter which is associated with H3K4me3, Pol-II, Ash2l, and Setd1a [15], which is verifying the non-TCF theory [5, 15]. Promoters are complex and it could be possible that additional elements might also play a key role (suggested in Figure 6). TERT and Axin2 might be regulated by non-TCF factors and also by TCF/Lef-1- $\beta$ catenin-dependent transcription, both at the same time (promoter add-on activation). While the first is a requirement for a TERT core transcription, the second enables a further increase (add-on), e.g. via Wnt3a or activated $\beta$-catenin MPCs, and this combinatorial activation was previously suggested [15]. Axin-2 seems similarly regulated in ESCs and iPSCs, indicating a dual role of non-TCF and TCF-factors in the promoter complexity. The schematization in Figure $6 \mathrm{~A}$ also reveals another factor, likely an EMT factor, like slug, twist, snail, miRNAs, or E-cadherin/ $\beta$-catenin-PTMs that might stem from E-cadherin regulation [20]. Unexpectedly, many of the important stemness factors are regulated by the combination of E-cadherin and non-TCF- $\beta$-catenin (see Figure 6: orange and blue circles in a blue cartridge; summarized in Figure $6 \mathrm{C}$ ). Also, there is another layer of a weaker regulation by $\beta$-catenin via TCF and a $\beta$-cateninPTM-isoform Figure 6; palm-color. This suggests a key role of E-cadherin and $\beta$-catenin in the stemness and pluripotency transcriptional network of iPSCs and ESCs two directly interacting factors in the pluripotency transcriptional network. Not only TERT but many additional $\beta$-catenin target genes seem to be also regulated by E-cadherin, like Rex-1, Sp-5, Bry, ESRRB, EphrinB2, EphA2, and the set of epiblast state markers like Oct-6, FGF-5 and many more (Fig. $6 \mathrm{C}$, blue circle regulated promoter target gene). $\beta$-catenin seems to be involved but in complex with cadherin at the membrane, i.a., and not with TCF or non-TCFs (Figure 2, S2, 5, 6). Lack 


\section{Journal of Embryology \& Stem Cell Research}

of $\beta$-catenin or E-cadherin both lead to a dysfunctional cell-adhesion of ESCs (Figure 2) and the EMT state [20] with a role on the transcriptional integrity of the stemness states. However, this state is usually only reached after the cells exit the metastable stemness network circuit and differentiate towards mesoderm in in a primitive streak like equivalent. The machinery of EMT factors [20] that is activated in early differentiation to mesorderm (Bry biomarker) is repressed by functional cell-adhesion complexes consisting of E-cadherin and $\beta$ catenin. Epithelial layer-like statics and signals of Ecadherin and membrane- $\beta$-catenin inhibit targets like Bry/T, Gelsolin, EMT-metastasis factors, and even TERT: combining a pluripotency and tumor-supressor function. In line with this, TERT is repressed by E-cadherin and activated by $\beta$-catenin in a Klf-4-dependent way. This might show that E-cadherin tumor-suppressor sequesters cancerogenic $\beta$-catenin that activates TERT - and assures the right TERT level in ESCs and iPSCs.
Using the four generalized pathway branches in a scalable systems biology and proof-of-principal approach, it would become more possible to reveal the complex situation on all of the affected genes and promoters that are directly or indirectly affected by $\beta$-catenin. The occurrence of the blue-yellow pattern in Figure 6, i.e. nonTCF and E-cadherin based promoter control, could have an interesting reason. How can a non-TCF-factor work in conjunction with an EMT factor downstream of Ecadherin? One explanation could be the role of E-cadherin signaling via PI3K/Akt or upregulation of EMT factors like slug via $\beta$-catenin $[20,32]$ or also without $\beta$-catenin. The role of E-cadherin signaling via PI3K/Akt has often been suggested but also in the cell-cycle, and slug and EMT [20] is not solely activated if $\beta$-catenin is activated; it only helps in a context- and EMT-dependent way (Supplementary Figure S6): but more research about this topic is still needed. THe marker changes in E-cadherin LOF ESCs indicate a role of an unknown $\beta$-catenin pathway on transcription.

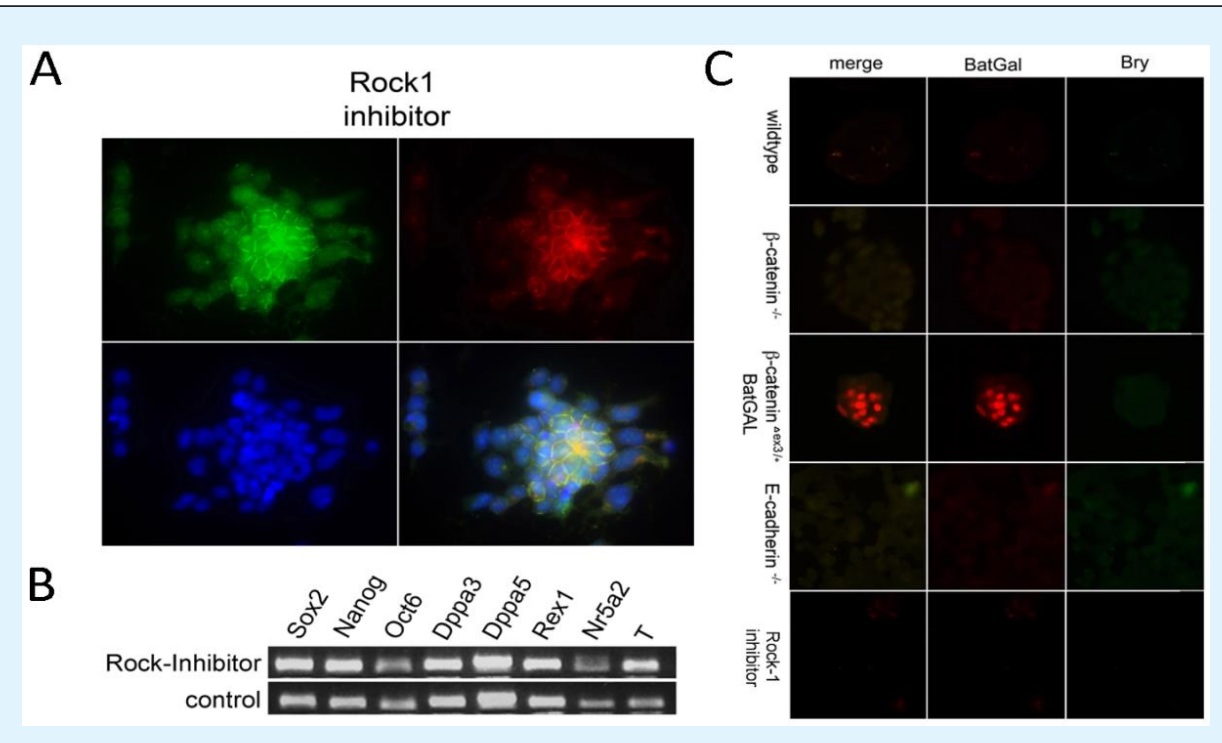

Figure S6: E-cadherin has a role in transcription of Bry/T that is independent of $\beta$-catenin and acts via EMT factors like Rock-1. Inhibition of rock increases Bry/- mRNA expression but not on the protein level.

The Bry/T promoter reveals some of its additional secrets of mesoderm regulation (see Figure 6): one regulatory element comprises TCF/Lef- 1 and a special PTM-isoform of $\beta$-catenin (palm-color), while a strong blockage or inhibition seems to be exerted by Ecadherin/ $\beta$-catenin at the same time (visualized as a blue circle). Hence, the combination of an indirect or direct "stop of E-cadherin repression" and "a $\beta$-catenin activation" is both driving Bry/T transcripts and there are multiple regulations on Bry/T mRNA and the protein level.
This combinatorial promoter regulation (Figure 6) explains the time and context dependent Bry-induction by Wnt-3a and $\beta$-catenin [5]. E-cadherin can use Rock singnaling, i.a., and Rock can mediate EMT in many systems [20]. Standard Rock-inhibition, for example, activates Bry/T-mRNA but not on the Bry/T protein level in the ESCs (Supplementary Figure S6) and can slightly affect the cytoskeleton, while plakoglobin is not very much helping to rescue the cell adhesion defect, at least in ESCs (see Figure 2, S3, S7). Context dependency of 


\section{Journal of Embryology \& Stem Cell Research}

activation seems to be controlled by a circuit of inductors, gatekeepers and transition checkpoints in stemness and differentiation, and in development and human diseases of tissue homeostasis. For example, E-cadherin gatekeeps
Bry and controls TERT, $\beta$-catenin gatekeeps the epiblast state with KLF-4 and Oct-3/4/Nanog/Sox-2 stemness factors.

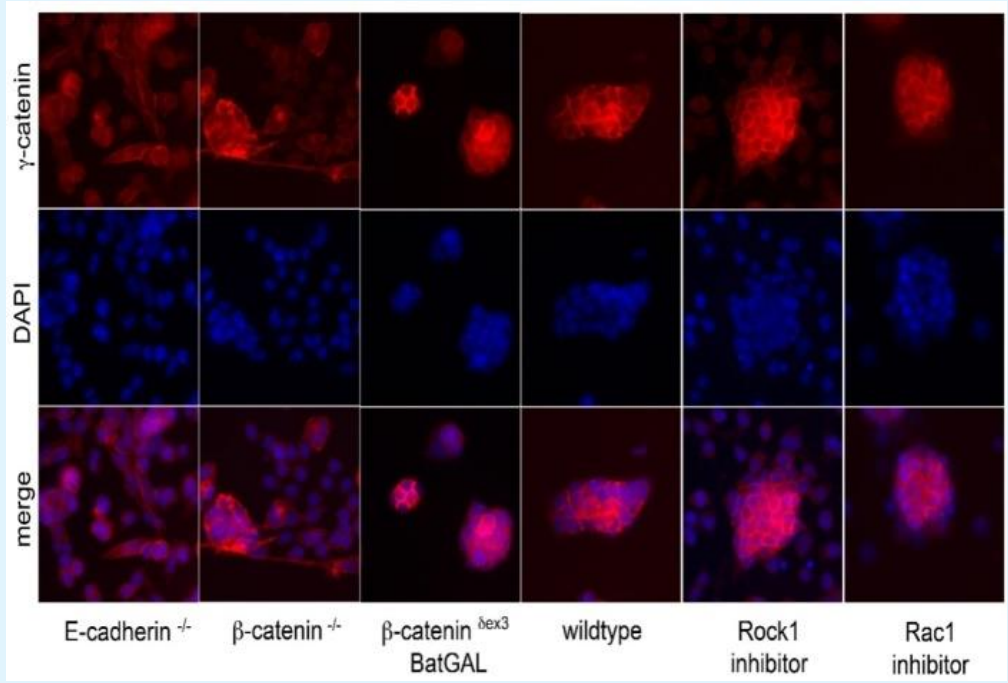

Figure S7: Plakoglobin (gamma-catenin) staining in the mutant ESCs reveals that it cannot much compensate for the loss of $\beta$-catenin in cell-adhesion: cell adhesion and its membrane localization is only marginal in ESCs and thus likely also in iPSCs.

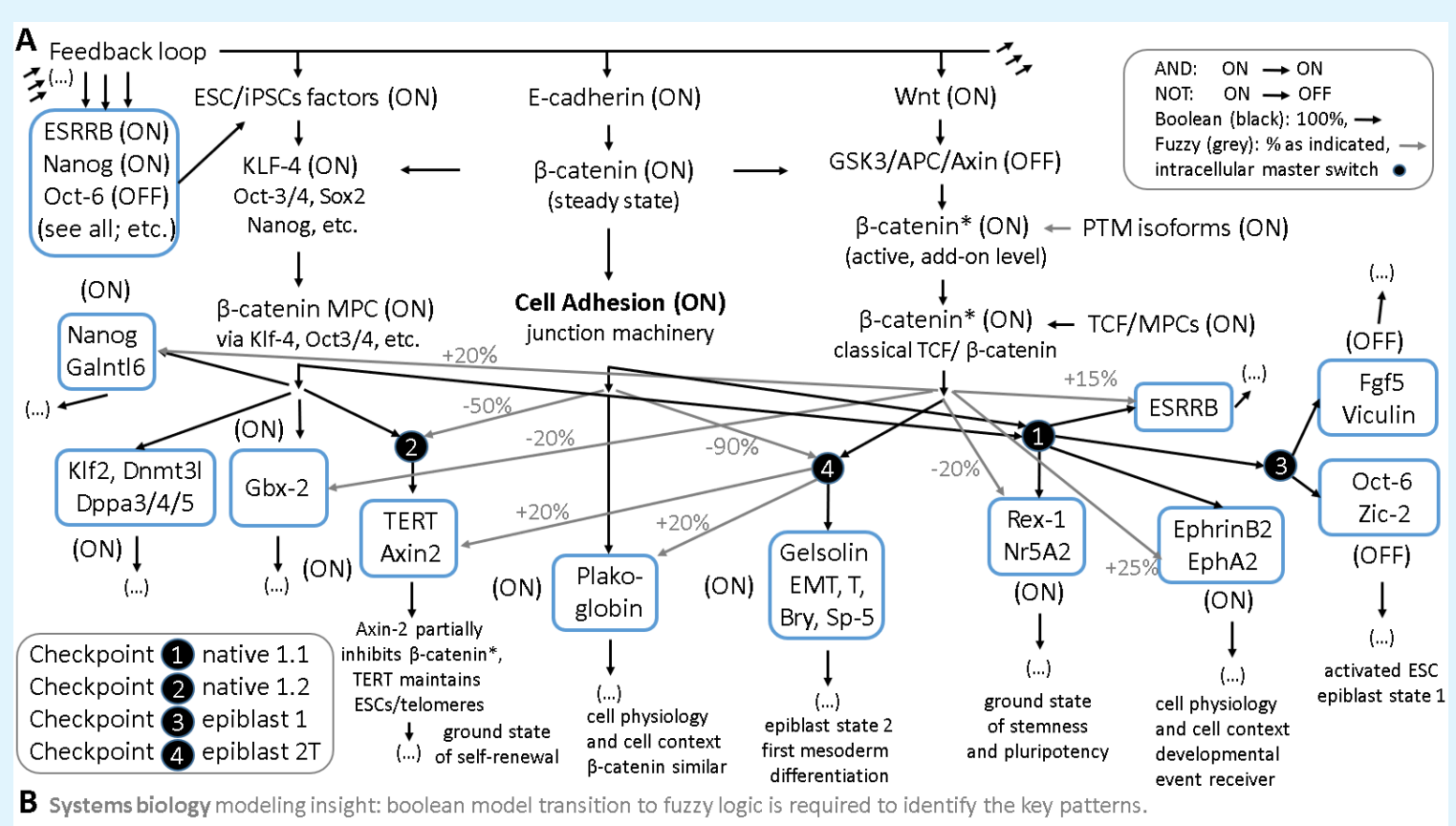

Figure 7: A boolean model of the stemness network introducing the new data and a transitioning to fuzzy logic. Four identified pathways and checkpoints mediate the native ground states of stemness, pluripotency, self-renewal, and the early transitioning states to epiblast $1+2$ comprising the early germ-layer differentiation to mesoderm (T). 


\section{Journal of Embryology \& Stem Cell Research}

Gelsolin and Sp-5, on the other hand, have a very similar expressional regulation, loss of E-cadherin activates them best, as an EMT signal triggers them and an epithelial Cdh-1 signal represses them. E-cadherin also lowers $\beta$-catenin protein but enhances its transcriptional activity, like the $\beta$-catenin-PTM-isoforms (Figure S2, 6 and 7).

The same discussion can be made accordingly for all other target genes (see Figure 6 and 7 and as previously mentioned). For example, Rex-1 and Nr5A2 are positively regulated by epithelial adhesion signals and a non-TCF- $\beta$ catenin complex, while the PTM-isoform and TCFs can have a slight negative effect. Does this combination of Ecadherin and non-TCF on many stemness promoters reveal a new mechanism of concerted action (blue and yellow circles) or the TCF and PTM-isoforms (red and green circles)? The key patterns of regulation are shown (Fig. 6).

At the TERT promoter, there is the specialty of a schematically vertical or inverse orientation, i.e., nonTCF- $\beta$-catenin transcriptional activation via Klf-4 that activates TERT [15], while an E-cadherin signal or effect that strongly represses a higher TERT mRNA level, which reveals a tumor-suppressor role of $\mathrm{Cdh}-1$ that represses metastasis, invasion, and immortalization, and hyperproliferation- even in self-renewing and pluripotent ESC and iPSCs that will switch of the stemness factors and TERT during differentiation (extrem levels of TERT could immortalize all of the cells, which might be prevented by E-cadherin). Interestingly, E-cadherin seems to manage the right protein level of TERT and stemness genes and is to be counted as a pluripotency factor like $\beta$-catenin that is usually expressed naturally in iPSCs and ESCs. Both, Ecadherin and $\beta$-catenin help to assure the right expression level of key target genes: together they might assure a target expression corridor assurance system. PTMsystems like GSK3 in Wnt signaling assure the right protein level of $\beta$-catenin and MPC funtion, while PTMisoforms of $\beta$-catenin can have different roles on the targets (Figure 6) and stemness and differentiation, for example, the trimethylation and acetylation of lysine 49 [23].

Are there any additional new mechanisms of $\beta$-catenin? On the mRNA and protein level, EIFs and EIF6 could be involved; Nanog protein is much in $\beta$-catenin and also in E-cadherin LOF ESCs (see Figure S2 A and B). EIF6 is involved in integrin binding at the membrane and dynamically elsewhere (Figure S4 C). Does a fundamental role of the regulation relay at the membrane, where also key branches of Wnt signaling and E-cadherin take their place [13]? Integrins and E-cadherin epithelial-signals could impact transcriptional outcomes with $\beta$-catenin in unseen ways involving $\beta$-catenin and starting from the membrane. Oct6/Fgf5/vinculin/Zic2 is repressed by the tandem and Klf2/Dnmt3l/Dppa3/4/5 only need a nonTCF/ $\beta$-catenin activation, maybe also Klf and Oct$3 / 4 /$ Nanog. Nanog is activated by non-TCF/ $\beta$-catenin and slightly by Tcf/ $\beta$-catenin/PTM isoforms, like is Galntl6. All regulations are summarized in Figure 6 and 7. Figure 6 finds $4+1$ effector pathways and regulatory effect patterns, while Figure 7 proposes 4 molecular checkpoints that require 4 transcriptional keys that direct the 4 mentioned transitioning states.

Finally, models will be very important to visualize all data in huge maps to allow future contributions in an ordered and systematic way. A preliminary and simple boolean stemness network model is given in Figure 7 and already reveals in its infancy that an extension into a system of fuzzy logic is needed to accommodate the modularity of the promoter and cellular factor complexity that happens at different quantities and that add-on activations and repressions could reach potential thresholds and different contexts that trigger the next stage and states of the differentiation program. This work also reveals an unexpected and radically misprized role and function of cell adhesion in stemness, ESCs and iPSCs. Cell-adhesion is also in play in stemness of ICM, iPSCs, and ESCs. Moreover, E-cadherin and $\beta$-catenin are key stemness and pluripotency factors in ESCs and iPSCs that drive the expression of very important factors of pluripotency. In the future, systems biology can further reveal how cell adhesion and cell-cell-contacts also play a crucial role in differentiation and stem cell maintenance in this and other settings and questions. Complexity makes a more systematic approach a new necessity and system biology is to be seen as an increasingly Complexity makes a more systematic approach a new necessity, and system biology is to be seen as an increasingly important, integrative and inclusive, service platform for all scientists. It shall be established as a free-access open science hub to promote the continuous progress and improvement in science in a more systematic and ordered approach that must also be fair and open for all - to enable all scientific contributors and to sustain the field of regenerative biology, stem cells, as an example that one shall bring free, open, and fair systems biology opportunities to all fields. 


\section{Journal of Embryology \& Stem Cell Research}

\section{Acknowledgments}

I am thankful and I acknowledge the research opportunity, including the material and cell line that was made available to me in my research project at the MaxPlanck-Institute for Immunology, and special thanks goes to Prof. Dr. Rolf Kemler for supervision and support.

\section{References}

1. Anton R (2016) on recent advances in human engineering. PLS - Cambridge University Press | CORE 35(2): 54-68.

2. Anton R, Kuhl M, Pandur P (2007) A molecular signature for the "master" heart cell. Bioessays 29(5): 422-426.

3. Orkin SH, Wang J, Kim J, Chu J, Rao S, (2008) The Transcriptional Network Controlling Pluripotency in ES Cells. Cold Spring Harb Symp Quant Biol 73: 95202.

4. Zhou Q, Chipperfield H, Melton DA, Wong WH (2007) A gene regulatory network in mouse embryonic stem cells. PNAS 104(42): 16438-16443.

5. Anton R, Kestler H, Kuhl M (2007) Beta-catenin signaling contributes to stemness and regulates early differentiation in murine embryonic stem cells. FEBS letters 581(27): 5247-5254.

6. Inoue $\mathrm{H}$, Nagata $\mathrm{N}$, Kurokawa H, Yamanaka S (2014) iPS cells: a game changer for future medicine. EMBO J 33(5): 409-417.

7. Ye-Ji S, Min-Seong K, Abee N, Ye-Jin Y, Su-Jindence K (2017) 2i Maintains a Naive Ground State in ESCs through Two Distinct Epigenetic Mechanisms. Stem Cell Reports 8(5): 1312-1328.

8. David PA (2004) Understanding the emergence of 'open science' institutions: functionalist economics in historical context. Industrial and Corporate Change 13(4): 571-589.

9. MacArthur BD, Ma'ayan A, Lemischka IR (2009) Systems biology of stem cell fate and cellular reprogramming. Nature Reviews Molecular Cell Biology 10: 672-681.

10. Basson MA (2012) Signaling in Cell Differentiation and Morphogenesis. Cold Spring Harb Perspect Biol 4(6): a008151.
11. Hatano SY, Tada M, Kimura H, Yamaguchi S, Kono T, et al. (2005) Pluripotential competence of cells associated with Nanog activity. Mech Dev 122(1): 6779.

12. Miyanari Y, Torres-Padilla ME (2012) Control of ground-state pluripotency by allelic regulation of Nanog. Nature 483(7390): 470-473.

13. Quaiser T, Anton R, Kuhl M (2006) Kinases and G proteins join the Wht receptor complex. Bioessays 28(4): 339-343.

14. Clevers H (2006) Wnt/beta-catenin signaling in development and disease. Cell 127(3): 469-80.

15. Hoffmeyer K, Raggioli A, Rudloff $\mathrm{S}$, Anton $\mathrm{R}$, Hierholzer A, et al. (2012) Wnt/ $\beta$-catenin signaling regulates telomerase in stem cells and cancer cells. Science 336(6088): 1549-1554.

16. Larue L, Ohsugi M, Hirchenhain J, Kemler R (1994) Ecadherin null mutant embryos fail to form a trophectoderm epithelium. Proc Natl Acad Sci U S A 122(17): 8263-8267.

17. Larue L, Antos C, Butz S, Huber O, Delmas V, et al. (1996) A role for cadherins in tissue formation. Development 122(10): 3185-3194.

18. Brault V, Moore R, Kutsch S, Ishibashi M, Rowitch DH, et al. (2001) Inactivation of the beta-catenin gene by Wnt1-Cre-mediated deletion results in dramatic brain malformation and failure of craniofacial development. Development 128(8): 1253-1264.

19. Chesbrough HW (2003) Open Innovation: the new imperative for creating and profiting from technology. Harvard Business Press (2003) ISBN 9781578518371.

20. Lamouille S, Xu J, Derynck R (2014) Molecular mechanisms of epithelial-mesenchymal transition. Nat Rev Mol Cell Biol 15(3): 178-196.

21. Lyashenko N, Winter $\mathrm{M}$, Migliorini $\mathrm{D}$, Biechele $\mathrm{T}$, Moon RT, et al. (2011) Differential requirement for the dual functions of $\beta$-catenin in embryonic stem cell self-renewal and germ layer formation. Nat Cell Biol 13(7): 753-761.

22. Davidson KC, Adams AM, Goodson JM, McDonald CE, Potter JC, et al. (2012) Wnt/ $\beta$-catenin signaling promotes differentiation, not self-renewal, of human 


\section{Journal of Embryology \& Stem Cell Research}

embryonic stem cells and is repressed by Oct4. Proc Natl Acad Sci U S A 109(12): 4485-4490.

23. Hoffmeyer K, Junghans D, Kanzler B, Kemler R (2017) Trimethylation and Acetylation of $\beta$-Catenin at Lysine 49 Represent Key Elements in ESC Pluripotency. Cell Rep 18(12): 2815-2824.

24. Ben-Shushan E, Thompson JR, Gudas LJ, Bergman (1998) Y Rex-1, a Gene Encoding a Transcription Factor Expressed in the Early Embryo, Is Regulated via Oct-3/4 and Oct-6 Binding to an Octamer Site and a Novel Protein, Rox-1, Binding to an Adjacent Site. Mol Cell Biol 18(4): 1866-1878.

25. Kondoh H (2018) Roles of ZIC-2 in regulation of pluripotent stem cells. Adv Exp Med Biol (1046): 339351.

26. Xu X, Guo M, Zhang N, Ye S (2018) Telomeric noncoding RNA promotes mouse embryonic stem cell self-renewal through inhibition of TCF3 activity. Am J Physiol Cell Physiol 314(6): C712-C720.

27. Huggins IJ, Bos T, Gaylord O, Jessen C, Lonquich B, et al. (2017) The WNT target SP5 negatively regulates WNT transcriptional programs in human pluripotent stem cells. Nat Commun 8(1): 1034.

28. Huang B, Deng S, Loo SY, Datta A, Yap YL, et al. (2016) Gelsolin-mediated activation of PI3K/Akt pathway is crucial for hepatocyte growth factor-induced cell scattering in gastric carcinoma. Oncotarget 7(18): 25391-25407.

29. Jing G, Wang Z, Zhuang X, He X, Wu H, et al. (2018) Suspended graphene oxide nanosheets maintain the self-renewal of mouse embryonic stem cells via down-regulating the expression of Vinculin. Biomaterials 171: 1-11.

30. Maekawa M, Yamaguchi K, Nakamura T, Shibukawa R, Kodanaka I, et al. (2011) Direct reprogramming of somatic cells is promoted by maternal transcription factor Glis1. Nature 474(7350): 225-229.

31. Aaronson Y, Livyatan I, Gokhman D, Meshorer E (2016) Systematic identification of gene family regulators in mouse and human embryonic stem cells. Nucleic Acids Res 44(9): 4080-4089.

32. Heo J, Lim J, Lee S, Jeong J, Kang H, Kim et al. (2017) Sirt1 Regulates DNA Methylation and Differentiation Potential of Embryonic Stem Cells by Antagonizing Dnmt3l. Cell Rep 18(8): 1930-1945.

33. Tang Y, Luo Y, Jiang Z, Ma Y, Lin CJ, et al. (2002) Jak/Stat3 signaling promotes somatic cell reprogramming by epigenetic regulation. Stem Cells 30(12): 2645-2656.

34. Baczynska D, Bombik I, Malicka-Blaszkiewicz M (2016) $\beta$-Catenin Expression Regulates Cell Migration of Human Colonic Adenocarcinoma Cells through Gelsolin. Anticancer Res 36(10): 5249-5256.

35. Huang TS, Li L, Moalim-Nour L, Jia D, Bai J, et al. (2015) Regulatory Network Involving $\beta$-Catenin, ecadherin, PI3k/Akt, and Slug Balances Self-Renewal and Differentiation of Human Pluripotent Stem Cells In Response to Wnt Signaling. Stem Cells 33(5): 14191433. 